\title{
ESTUDIO INTRASITIO DE UN YACIMIENTO ARCAICO CON ARQUITECTURA EN LA COSTA DE TALTAL, DESIERTO DE ATACAMA, NORTE DE CHILE
}

\author{
INSTRASITE STUDY OF AN ARCHAIC SETTLEMENT WITH ARCHITECTURE ON \\ THE COAST OF TALTAL, ATACAMA DESERT, NORTHERN CHILE
}

\author{
Ximena Power ${ }^{1}$ y Diego Salazar ${ }^{2}$
}

\begin{abstract}
El surgimiento y proliferación de arquitectura doméstica y funeraria en la costa desértica de Atacama constituye uno de los procesos sociales con mayor atención en la historia de la investigación del Norte Grande de Chile, siendo este un fenómeno que transcurre en un momento acotado de la secuencia prehispánica de la zona (ca. 5700-4000 cal. AP). A fin de profundizar en las particularidades de este periodo, se presenta el estudio intrasitio de Caleta Bandurrias, ubicado en el tramo costero de Taltal-Paposo. Desde el análisis de distintas líneas de evidencia, se discuten las actividades desarrolladas en el yacimiento, así como los patrones de subsistencia y la organización tecnológica de los cazadores recolectores pescadores que lo habitaron. Los resultados obtenidos y su consecuente evaluación en el marco del patrón de asentamiento regional, sugieren el uso residencialfunerario y/o de agregación social del sitio, así como un modo particular de aproximación al litoral mediante una estrategia de movilidad residencial coordinada en torno a la disponibilidad de recursos locales y a dichos espacios construidos.
\end{abstract}

Palabras claves: cazadores recolectores pescadores, arquitectura funeraria, campamento residencial, costa del desierto de Atacama.

The emergence and proliferation of domestic and funerary constructions on the Atacama Desert coast, which takes place for a short time of the pre-Hispanic period of the region (ca. 5700-4000 cal. BP), is a social process and architectural phenomenon that attracts much of the attention of the archaeological research of Northern Chile. To delve into the unique characteristics of this period, an intrasite study of the Caleta Bandurrias site (Taltal) is presented. The analysis of different lines of evidence suggests activities, subsistence patterns, and technological organization of local hunter gatherer fishers inhabiting the area. The results, and their subsequent analysis within a context of regional settlement, suggest a residential-funerary use and/or social aggregation function of the site, as well as a particular type of advance towards the coast through a residential mobility strategy based on the availability of local resources and built environment.

Key words: Hunter gatherer fishers, funerary architecture, residential camp, Atacama Desert coast.

Durante el rango temporal comprendido entre los ca. 5700-4000 años AP, las comunidades del litoral de arreismo absoluto transformaron su espacio de vida mediante la construcción de estructuras circulares de piedra, las cuales constituyen a la fecha una de las primeras evidencias arquitectónicas y funerarias formales para el periodo Arcaico en la costa del Norte Grande de Chile (Llagostera 1989, 2005; Núñez y Santoro 2011; Urbina et al. 2011). La particularidad y homogeneidad de este patrón constructivo-funerario, llamó tempranamente la atención de los primeros investigadores de la costa del desierto de Atacama, quienes asociaron este fenómeno a "la gente de los círculos de piedra" (Capdeville 1921b; Mostny 1964) o a "las poblaciones marítimas con arquitectura" (Schaedel 1957). Es durante la década de 1970 que se inaugura la investigación sistemática de este tipo de yacimientos, estableciéndose como referente el sitio Caleta Huelén 42, ubicado en la desembocadura del Río Loa (Núñez, L. 1971; Núñez, P. 1974; Núñez et al. 1974; Núñez y Santoro 2011; Zlatar 1983, 1987).

Actualmente, un gran cúmulo de trabajos permiten discutir las particularidades de este momento en la secuencia prehispánica de distintas localidades del litoral arreico entre el Río Loa y Chañaral, registro que incluye manifestaciones arquitectónicas en Tocopilla

\footnotetext{
${ }^{1}$ Programa de Doctorado en Antropología UCN-UTA, Universidad Católica del Norte, San Pedro de Atacama, Chile. xpv001@alumnos.ucn.cl

${ }^{2}$ Departamento de Antropología, Facultad de Ciencias Sociales, Universidad de Chile, Santiago, Chile. dsalazar@uchile.cl
} 
(Salazar et al. 2010;), Cobija (Andrade et al. 2016; Bittmann 1984; Castro et al. 2012, 2016; Montenegro 1981; Olguín et al. 2015a), Michilla (Ballester et al. 2018), Mejillones-Antofagasta (Ballester et al. 2014b, 2014a; Bustos 1974; Cruz y Llagostera 2011; Llagostera 1989), Paposo-Taltal (Capdeville 1921b; Castelleti 2007; Contreras et al. 2007, 2008, 2011; Latcham 1939; Llagostera y Llagostera 2010; Mostny 1964; Power 2015; Salazar et al. 2015) y Chañaral (Salazar et al. 2015) (Figura 1). A su vez, propuestas de síntesis permiten ponderar las evidencias a nivel regional, avanzando hacia la comprensión de los patrones de asentamiento, estrategias de movilidad y redes de interacción desarrolladas por las comunidades costeras de este periodo (Ballester y Gallardo 2011; Ballester et al. 2017; Bittmann y Munizaga 1984; Castelleti 2007; Castro et al. 2012; Llagostera 2005, 2013; Núñez 1984; Núñez y Santoro 2011; Salazar et al. 2015). En términos generales, las investigaciones coinciden en señalar el uso residencial y funerario de estos asentamientos, interpretados a lo largo de la historia de la investigación como cementerios (Capdeville 1921b; Mostny 1964), aldeas (Bittman 1984; Bustos 1974; Contreras et al. 2007, 2008, 2011; Núñez 1974; Núñez et al. 1974; Núñez 1984; Núñez y Santoro 2011) o, más comúnmente, como campamentos residenciales semipermanentes (Ballester y Gallardo 2011; Ballester et al. 2014b, 2014a, 2017, 2018; Castelleti 2007; Castro et al. 2012, 2016; Olguín et al. 2015a; Salazar et al. 2015; Zlatar 1983, 1987).
A fin de aportar antecedentes a la problemática ocupacional de los yacimientos de este periodo y las consecuentes transformaciones en la costa atacameña, se presenta el estudio del sitio Caleta Bandurrias, ubicado en el tramo litoral Taltal-Paposo. El trabajo se erige como un ejercicio de evaluación de indicadores de funcionalidad de sitios a partir de distintas líneas de evidencia, con el objetivo de reconstruir la historia ocupacional del yacimiento, las actividades desarrolladas y los rangos de movilidad de las poblaciones costeras que ocuparon la localidad durante el denominado periodo Arcaico Tardío (Castelleti 2007). Los resultados se ponderan en relación a los sitios y a las distintas propuestas de movilidad y asentamiento sugeridas para el periodo a nivel regional. Se propone el uso residencial del yacimiento en base a la disponibilidad de recursos locales y a la red de espacios construidos en el tramo costero de la zona, a la vez que se discuten evidencias que permiten formular nuevas hipótesis sobre el uso de este sitio, a ser evaluadas en futuras investigaciones.

\section{Contexto Geográfico}

La costa del Norte Grande de Chile ha sido subdividida en distintas áreas en base a sus diferencias geomorfológicas y ecológicas. La zona ubicada entre Pisagua y Chañaral, comprende lo que ha sido denominado comúnmente como litoral árido de arreismo absoluto (Llagostera 1989, 2005). Esta subárea de la costa del Pacífico está desconectada de los corrientes fluviales

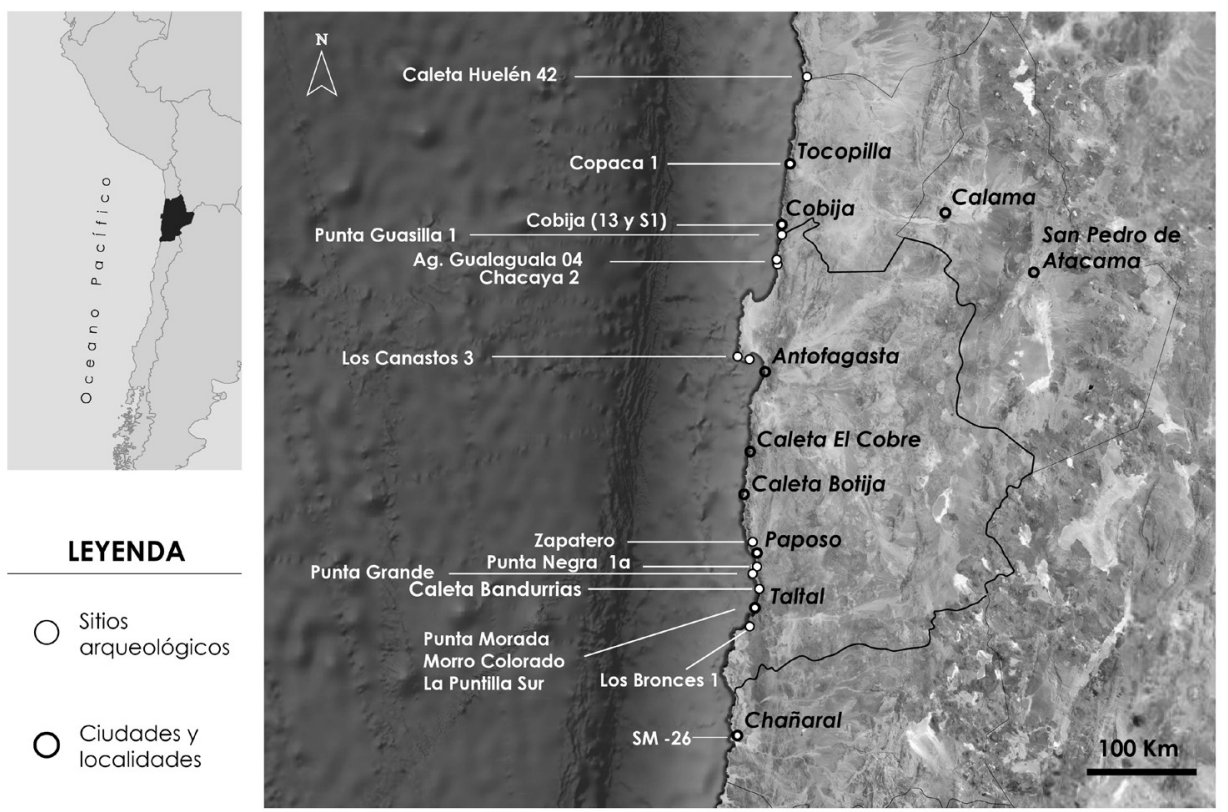

Figura 1. Sitios arqueológicos con arquitectura Caleta Huelén-42 referidos en el texto.

Archaeological sites with Caleta Huelén-42 architecture mentioned in the text. 
de la cordillera de los Andes, siendo el Río Loa el único afluente con cauce permanente en casi $800 \mathrm{~km}$. A pesar de esto, existen diversos afloramientos de agua ubicados en la plataforma litoral y en la Cordillera de la Costa, alimentados principalmente por precipitaciones costeras asociadas a la anomalía climática El Niño (ENSO) (Herrera y Custodio 2014; Herrera et al. 2018); recursos hidrológicos que constituyeron una variable esencial en el asentamiento humano del desierto costero (Llagostera 1989, 2005, 2013; Núñez y Varela 1967-68).

En contraste con dichas condiciones hiperáridas, el océano Pacífico que baña estas costas constituye uno de los ecosistemas marinos más ricos del mundo. Dotado de una amplia gama de recursos bióticos producto del efecto de la corriente de Humboldt y la surgencia costera (Thiel et al. 2002), el mar conformó el principal foco de explotación económica de las antiguas poblaciones litorales (Llagostera 1989, 2005). Complementariamente, los faldeos occidentales de la Cordillera de la Costa ofrecen recursos terrestres asociados al ecosistema de lomas y a aguadas, los cuales se componen de una gran diversidad de especies vegetales con potencialidad de uso como alimento, combustible y materia prima (Gutiérrez y Lazo 1996), así como diversas especies de fauna tales como guanacos, zorros, aves, reptiles y roedores (Marquet et al. 1998).

La localidad de Taltal se ubica en el segmento meridional de este litoral arreico. Se enmarca en la
Bahía de Nuestra Señora, en una estrecha plataforma litoral que limita al este con la Cordillera de la Costa, la que alcanza hasta 2.000 metros de altura. La fachada occidental del cordón montañoso presenta quebradas rocosas empinadas y una plataforma litoral constreñida. Características de este relieve son las puntas rocosas o puntillas que se extienden desde el farellón y que forman bahías rocosas de escasa profundidad. La localidad presenta características particulares para el asentamiento humano, dada la existencia de más de 40 aguadas desde Caleta Botija hasta Taltal. Dichos manantiales afloran principalmente en la base de las quebradas y en la planicie costera, posibilitando su uso local, tal como atestigua la directa relación de estos recursos con los asentamientos prehispánicos de la zona (Borie y Soto 2011; Contreras et al. 2007, 2011; Núñez y Varela 1967-68).

\section{El Sitio Caleta Bandurrias. Emplazamiento y Sectorización}

Caleta Bandurrias corresponde a una bahía rocosa semi-arenosa ubicada al norte de la desembocadura de la quebrada y de la puntilla homónima, a unos 20 $\mathrm{km}$ al norte de Taltal (Figura 2a). En los alrededores, se observan al menos cuatro islotes que actualmente sirven de apostaderos de lobos marinos y densas colonias de aves, así como tres aguadas: "Bandurrias", "Estancia Vieja" y "Cachinales" (Contreras et al. 2007).
A

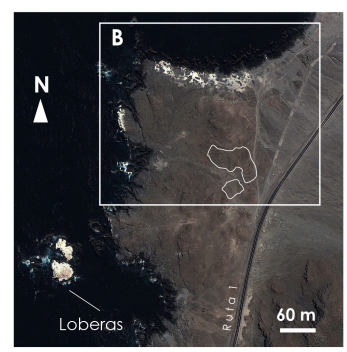

B

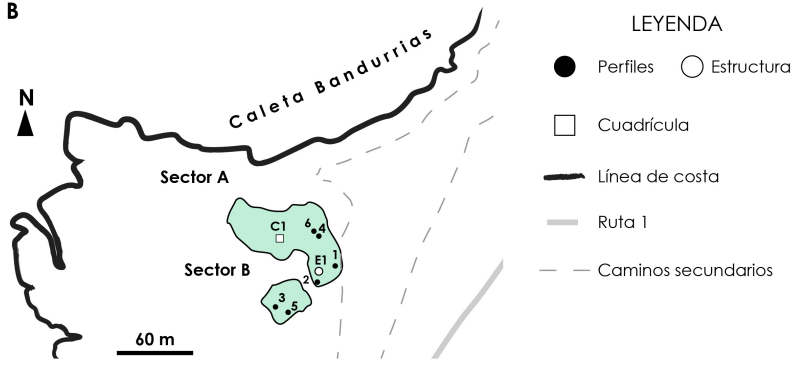

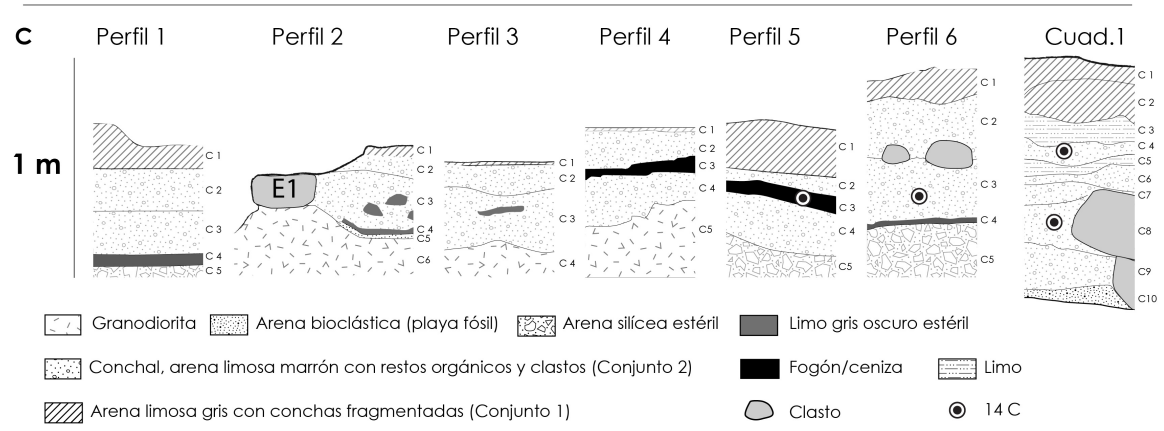

Figura 2. El sitio Caleta Bandurrias. a. Emplazamiento; b. Sectores; c. Secuencia estratigráfica de las unidades de control. Caleta Bandurrias site. a. Location; b. Site areas; c. Stratigraphic sequence of control units. 
La caleta es utilizada actualmente como área de manejo para la explotación de recursos marítimos por orilleros, pescadores y buzos artesanales.

Los primeros trabajos arqueológicos del área fueron realizados por Augusto Capdeville, quien junto a Manuel González y Belisario Flores constataron la existencia de diversos yacimientos (Capdeville 1920). Destaca el reporte de un collar, puntas pedunculadas, una hoja bifacial y pesas líticas recuperadas de excavaciones en Caleta Bandurrias (cf. Soto et al. 2018:Figura 2), materialidad distintiva de la "Civilización Dolménica" postulada por el autor (Capdeville 1921b; Mostny 1964).

El sitio Caleta Bandurrias, también denominado Bandurrias-1 (Contreras et al. 2007, 2008, 2011; Núñez y Santoro 2011), se compone de dos concentraciones tipo conchal dispuestas sobre dos terrazas marinas pleistocénicas sin clara solución de continuidad (Guendon 2009) (Figura 2b). El sector A $\left(6.389 \mathrm{~m}^{2}\right)$ se ubica sobre la terraza de +10 msm y se extiende desde la ladera de un afloramiento batolítico hasta el borde de la terraza, registrando abundantes bloques de estructuras colapsadas y un consistente volumen depositacional (= $70 \mathrm{~cm}$ ). El sector B, en tanto, se ubica en la terraza marina de $+20 \mathrm{msm}$, conformando un conchal más acotado $\left(1.648 \mathrm{~m}^{2}\right)$, con una menor cantidad de clastos en superficie y una menor potencia estratigráfica $(=50 \mathrm{~cm})$. Ambos sectores presentan severos signos de destrucción por saqueo, maquinaria pesada y caminos pedestres $\mathrm{y}$ vehiculares de uso vigente.

\section{Metodología}

Las actividades de investigación realizadas en el marco de los proyectos Fondecyt 1080666 y 1151203 , se orientaron tanto al registro superficial como estratigráfico del yacimiento. El registro de superficie consideró la definición del área de extensión y sectorización del sitio, recolección de material diagnóstico y la cuantificación de bloques de muros colapsados expuestos. A fin de lograr una estimación aproximada del número mínimo de estructuras en el sitio, la cifra obtenida de la cuantificación de bloques, se dividió por la cantidad de bloques promedio que componen las estructuras "tipo Caleta Huelén 42" ( $\mathrm{N}=50$; cf. Power 2015). Los estudios estratigráficos, por su parte, consideraron la excavación de 19 pozos de sondeos de 0,5 x 0,5 m en distintos sectores del sitio. Asimismo, se analizaron secuencias estratigráficas de siete perfiles expuestos, desde los cuales se obtuvieron dos columnas de muestreo $(0,5 \times 0,5 \mathrm{~m})$ y muestras de datación (Figura 2c). Adicionalmente, se excavó una unidad de 1x $1 \mathrm{~m}$ (Cuadrícula-1) y el interior de un recinto arquitectónico (Estructura-1). Una vez expuesto completamente el muro de la Estructura-1, se realizó su registro arquitectónico en base a protocolo directriz (Castro et al. 1993).
El análisis de los materiales recuperados de los contextos fechados incluyó estudios arqueozoológicos dirigidos a la determinación anatómica y taxonómica mediante claves propuestas para invertebrados marinos (Guzmán et al. 1998; Marincovich 1973, 1992; Osorio 2002;Zúñiga 2002), peces (Falabellaetal. 1995; Lamillay Bustamante 2005; Nakamura 1985), camélidos (Pacheco Torres et al. 1994; Sierpe 2015); cetáceos (Buchholtz y Schur 2004; Kasuya 1973) y roedores (Reise 1973). La abundancia relativa de los invertebrados consideró el cálculo de MNI en base a elementos anatómicos no repetibles (Mason et al. 1998; Claassen 1998). Para el conjunto osteofaunístico se consideró NISP y MNI; a la vez que MNE, MAU y \%MAU, para la representación de partes esqueletarias (Binford 1981; Grayson 1984; Lyman 2008). La estimación de la composición etárea se realizó a partir de la longitud peristomal de valvas completas de Concholepas concholepas (Báez et al. 2004; Jerardino et al. 1992) y en base a criterios de tamaño y grado de fusión de epífisis de diversos elementos anatómicos de guanacos (Kaufmann 2004) y pinnípedos (Borella et al. 2013).

El análisis del conjunto artefactual consistió en una caracterización macroscópica de artefactos líticos, conquiológicos y óseos. Para artefactos formatizados, se realizó una caracterización de la morfología general y de bordes o superficie(s) activa(s), materia prima, forma base, técnica de modificación y atributos métricos, entre otros (Andrefsky 1998; Bate 1971; Santander 2011; Soto et al. 2018). El análisis de derivados de talla consideró completitud, materia prima, tipo de derivado, talón, índice de corteza, dimensiones, determinación de número de total de piezas $(\mathrm{N})$ y número mínimo de derivados (NMD) (Andrefsky 1998).

\section{Resultados}

\section{Secuencia estratigráfica}

Los estudios realizados permitieron esbozar una secuencia ocupacional sintética para el sitio Caleta Bandurrias caracterizada por los siguientes conjuntos arqueoestratigráficos:

Conjunto 1: estratos superficiales de escasa potencia y posiblemente re-trabajados, de baja compactación, color grisáceo, con alto contenido de conchas fragmentadas y presencia de fragmentos cerámicos y de objetos de metal (Figura 2c). Se estima que este conjunto correspondería al periodo Intermedio Tardío, en acuerdo a los tipos cerámicos registrados (Varela 2009), así como ocupaciones más recientes de carácter histórico y subactual.

Conjunto 1(2): conjunto acerámico subyacente al conjunto 1 , caracterizado por estratos poco potentes de 
conchal, alternados con depósitos de arenas silíceas y/o limo, con baja frecuencia de material cultural y ecofactual. Este conjunto se localiza únicamente en el margen poniente del Sector A y no cuenta actualmente con fechados.

Conjunto 2: grupo acerámico definido por densos estratos de conchal, fogones, depósitos de ceniza y bloques y/o estructuras (Figura 2c). Este conjunto es el más extenso vertical y horizontalmente, representándose en ambos sectores del yacimiento y corresponde al primer nivel ocupacional del sitio, definiendo el momento de construcción de las estructuras y la conformación de los basurales. Los fechados obtenidos establecen un rango de ca. 5800- 4600 años cal. AP para este conjunto, siendo atribuible al periodo Arcaico Tardío o Arcaico IV, según las últimas propuestas cronológicas locales (Castelleti 2007; Salazar et al. 2015) (Tabla 1).

\section{Características arquitectónicas y secuencia de construcción}

Los numerosos bloques líticos expuestos en la superficie del sitio Caleta Bandurrias atestiguan la pretérita existencia de construcciones arquitectónicas. Se registró un total de 1288 bloques graníticos redondeados, originalmente disponibles en la playa fósil de $+10 \mathrm{~m}$ sobre la que se emplaza el sitio. De dicha cuantificación, considerando igualmente a la Estructura-1 ( $\mathrm{N}=48$ bloques), se estimó un mínimo de 27 estructuras para el sitio, cantidad aproximativa debido a la existencia de un número indeterminado de bloques bajo la superficie actual y a la variabilidad intrínseca de cada muro.
La Estructura-1 constituye el único ejemplar arquitectónico completo registrado hasta la fecha en el sitio (Figura 3). Específicamente, se trata de un recinto de planta circular (2,57 x 2,3 m), conformado por un muro continuo de hilada simple dispuesto sobre el borde de una depresión natural del sustrato rocoso. Los depósitos intramuro revelaron una secuencia compuesta por dos unidades arqueoestratigráficas, evidenciándose severas alteraciones por huaqueo en el primer estrato (Figura 4a). El segundo conjunto se compone de una matriz limo-arenosa de coloración rojiza -posiblemente por la agregación de pigmentos de óxido de hierro-, dispuesta inmediatamente sobre el sustrato rocoso de granodiorita. El sustrato presenta dos fosas antrópicas, dentro de las cuales fueron recuperados restos óseos humanos desarticulados teñidos de rojo, diversas piezas líticas bifaciales y cuentas de concha ${ }^{1}$ (Figura 4a).

La lectura estratigráfica del perfil 2 , al exterior de la Estructura-1, permitió develar la secuencia constructiva de esta última (Figura 4a). En primera instancia, se constató la modificación antrópica de la topografía original del sustrato mediante la percusión y excavación de la roca. Dicho procedimiento, permitió habilitar dos fosas en la planta del recinto y acondicionar la base perimetral del mismo para levantar el muro. Consecutivamente, la mampostería se realizó con bloques graníticos de playa sin modificación, conformándose diferentes secciones del muro en acuerdo a la modalidad de aparejo (Figura 4b). Los estratos registrados al exterior del muro, si bien son posteriores a la construcción, plantean dos alternativas para su formación: un relleno sedimentario intencional durante la etapa constructiva; alternativamente, una depositación progresiva resultado del uso del recinto. $\mathrm{Al}$ no contar con fechados de dichos estratos, la disposición

Tabla 1. Fechados 14C del sitio Caleta Bandurrias calibrados con dos sigmas utilizando la curva SHCal 13 (Hogg et al. 2013), mediante Oxcal 4.3 (Bronk Ramsey y Lee 2013). Las muestras bioantropológicas y de concha fueron corregidas por efecto reservorio en acuerdo a valores propuestos por Ortlieb et al. (2010).

$14 C$ dates from the Caleta Bandurrias site calibrated with two sigma, using the SHCal 13 curve (Hogg et al. 2013) through Oxcal 4.3 (Bronk Ramsey and Lee 2013). Bioanthropological and shell samples were corrected by reservoir effect according to values proposed by Ortlieb et al. (2010).

\begin{tabular}{ccccc}
\hline ID Laboratorio & Muestra & Material & $\begin{array}{c}\text { Edad 14C } \\
\text { (años AP) }\end{array}$ & $\begin{array}{c}14 \mathrm{C} 2 \sigma \\
\text { Edad cal. } \\
\text { (añosAP) }\end{array}$ \\
\hline BETA-290983 & CB- P6 - C3 & concha & $5430 \pm 60$ & $5833-5306$ \\
\hline BETA-290982 & CB- P5- C3 & concha & $5180 \pm 70$ & $5569-4951$ \\
\hline BETA-290981 & CB- Estr- 1 & óseo (bioantrop.) & $4920 \pm 30$ & $5265-4685$ \\
\hline UGAMS 12055 & CB- C1- C8 & óseo (camélido) & $4810 \pm 25$ & $5590-5332$ \\
\hline UGAMS 12053 & CB- C1- C4 & óseo (camélido) & $4430 \pm 40$ & $5270-4849$ \\
\hline BETA-255685 & CB- C1- C4 & carbón & $4340 \pm 40$ & $5033-4726$ \\
\hline
\end{tabular}




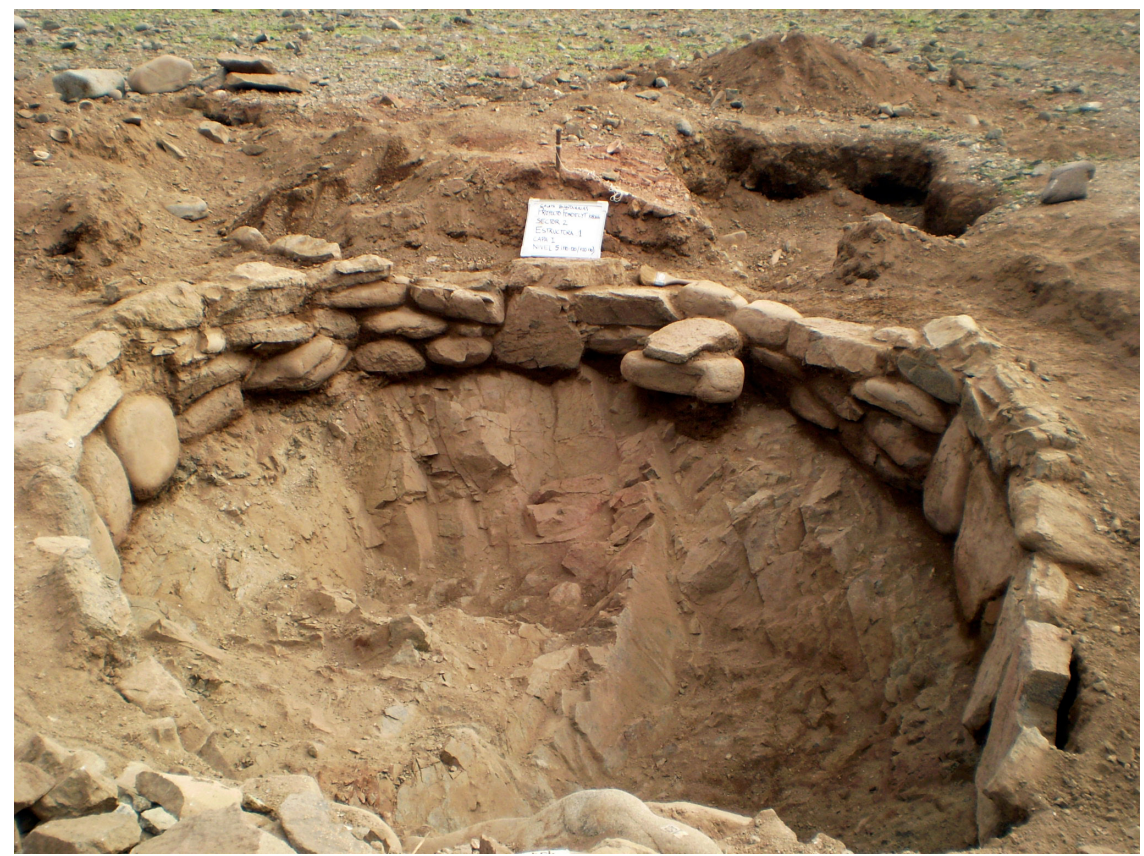

Figura 3. Estructura-1.

Structure-1.

a.

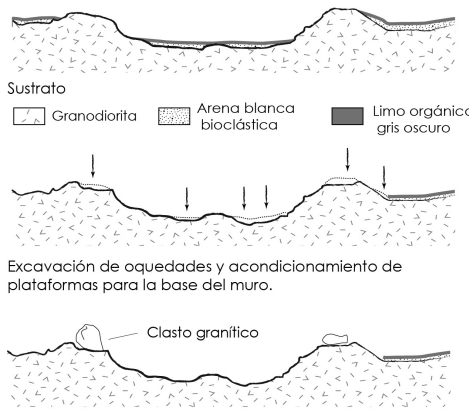

Mampostería: aplomo de clastos y conformación del muro.

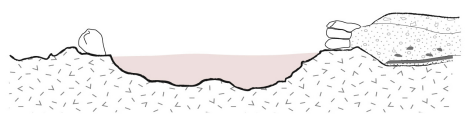

Formación del piso y sedimentación extramuro
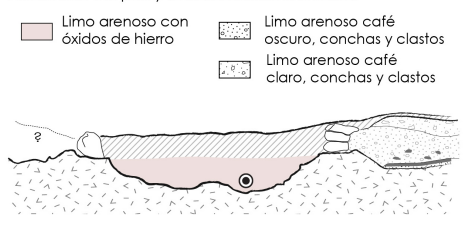

Abandono y relleno de la estructura "VII//. Capa superficial b.
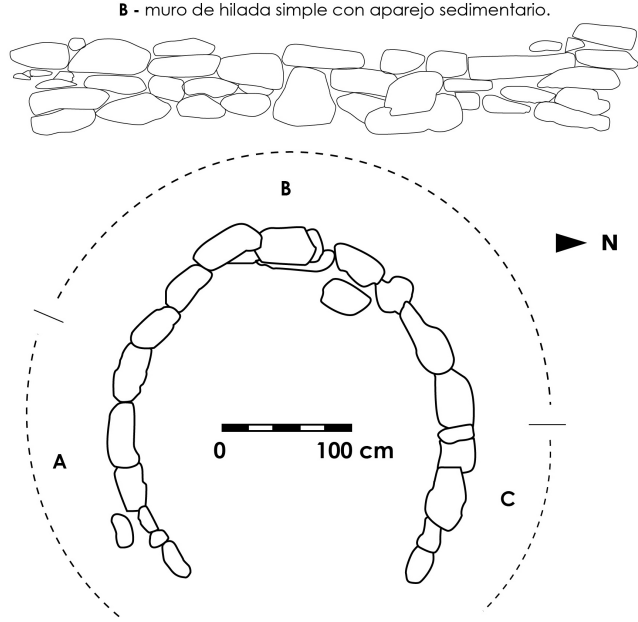

A - muro de hilada simple con aparejo vertical y horizontal de clastos.

C - muro de hilada simple con aparejo vertical y sedimentario de clastos.
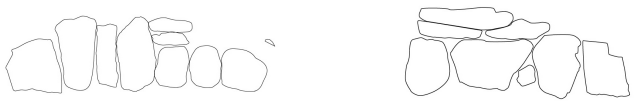

Figura 4. Características constructivas de la Estructura-1. a. Secuencia estratigráfica; b. Planta arquitectónica y secciones del muro. Constructive characteristics of Structure-1. a. Stratigraphic sequence; $b$. Floor plan and sections of the wall. 
aérea o semisubterránea de la estructura no pudo ser precisada.

\section{Aprovisionamiento, procesamiento y descarte de recursos faunísticos}

\section{Invertebrados marinos}

Se reconocieron 28 taxa a nivel de especie, destacando entre los gastrópodos los fisurélidos (27\%),
Tegula atra (21\%) y Concholepas concholepas $(18 \%)$ (Tabla 2). El grupo de los poliplacóforos es igualmente considerable, representándose mayoritariamente Acanthopleura echinata (15\%). Los especímenes señalados habitan el intermareal rocoso expuesto y zonas submareales de escasa profundidad (i.e. pozas de roqueríos o playas de guijarros), constituyendo espacios con buena accesibilidad en el medio litoral adyacente al sitio(Camus et al. 2012; Guzmán et al. 1998; Marincovich 1973; Osorio 2002; Otaiza y Santelices 1985). La

Tabla 2. Restos identificados de invertebrados marinos en Caleta Bandurrias. Remains of invertebrate shell identified at Caleta Bandurrias.

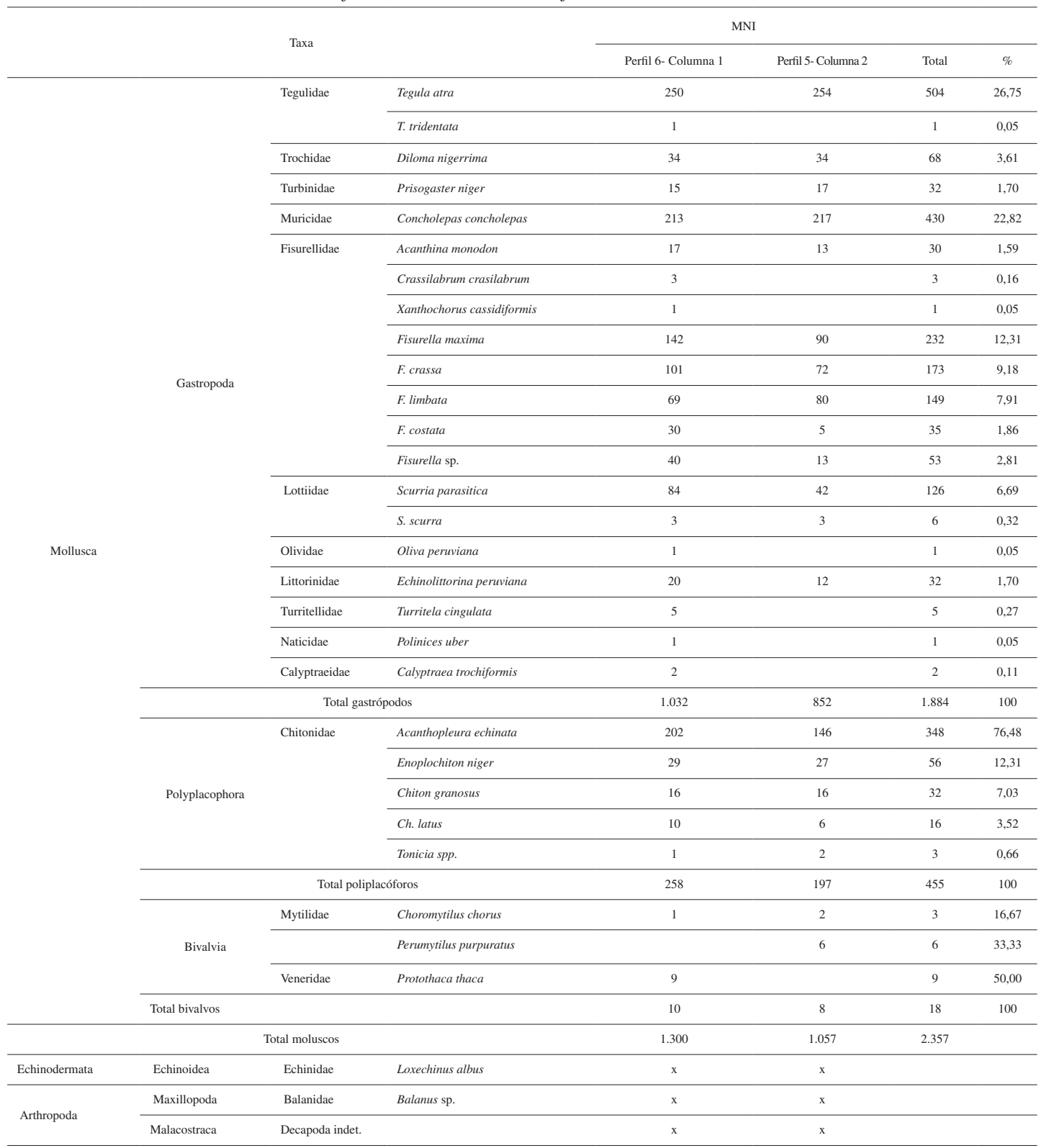


distribución de tallas de Concholepas concholepas confirma la explotación de dicha franja litoral, en cuanto representan a especímenes en etapa de primera madurez reproductiva (Báez et al. 2004) (Figura 5). La presencia de dichas especies, se relaciona con la captura manual de moluscos con alta biomasa y el transporte de los individuos completos hacia el sitio para su respectivo desconche y consumo (Waselkov 1987).

Por su parte, la presencia marginal de moluscos de menor rendimiento se plantea en el marco de la recolección conjunta con otros recursos marinos (Tabla 2). Dichos taxa cohabitan con especies de mayor ubicuidad (Diloma nigerrima, Prisogaster niger, Echinolittorina peruviana, Perumytilus purpuratus), son epibiontes de otros organismos (Crepidula dilatata, Scurria parasitica, Balanus spp.) o se adosan a estipes de algas (Scurria scurra) (Guzmán et al. 1998; Marincovich 1973). En tanto, la baja frecuencia de bivalvos, como Protothaca thaca, indica el acceso a bancos de arena localizados en el medio litoral aledaño o de playas cercanas (p.ej., Cachinales, a $2 \mathrm{~km}$ ); mientras que Choromytilus chorus se dispone en la franja submareal a 4-20 metros de profundidad (Guzmán et al. 1998), lo cual señala la inmersión submarina como requerimiento para su extracción, sin descartarse la recolección de valvas vacías en el borde litoral.

\section{Peces}

Los especímenes identificados comprenden 13 taxa a nivel de especie, una a nivel de clase (Chondrichthyes) y una a nivel de género (Genypterus) (Tabla 3). Destaca la abundancia del jurel Trachurus murphyi, tendencia que se manifiesta en todas las unidades de control. Los taxa con subsecuente representatividad numérica ( $>1 \%$ NISP) corresponden a Paralichthys microps, Semicossyphus darwini, condrictios, Xiphias gladius y Cilus gilberti. El registro de elementos de cráneo y postcráneo para la mayoría de los taxa identificados apoya el ingreso de especímenes completos al sitio.

En base al hábitat de las especies identificadas y a literatura referencial de la zona, se proponen métodos de captura en orilla y aguas afuera (sensu Béarez et al. 2016). De este modo, las especies bentodemersales (i.e. $P$. microps, S. darwini, C. gilberti, G. nigra, A. punctatus, Ch. variegatus, $P$. jugularis) estarían disponibles en medios rocosos de baja profundidad, lo cual sugiere una pesca preferentemente en base a línea simple y anzuelo, métodos de enmalle, arponeo o captación manual desde la costa (Ballester 2017; Contreras 2010). Por otra parte, la captura de especies pelágicas (i.e. T. murphyi, E. maculatum y T. atun) se habría realizado con redes, línea de mano y/o espinel a escasa profundidad, ya fuese en aguas retiradas de la costa o en orilla, dependiendo del periodo específico de su ciclo migratorio (Ballester 2017; Béarez et al. 2016; Castro et al. 2016; Rebolledo et al. 2016). En tanto, la caza de Xiphias gladius se realizaría con arpones vía embarcaciones posiblemente en aguas retiradas de la costa (Ballester 2017, 2018; Ballester y Gallardo 2011; Béarez et al. 2016; Castro et al. 2016; Escobar 2017; Olguín et al. 2014, 2015a; entre otros).

\section{Mamíferos marinos y terrestres}

La muestra registró un bajoíndice de meteorización, aunque el alto grado de fragmentación (78\%) limitó cuantiosamente el potencial de reconocimiento. El conjunto de especímenes identificados, se compone mayoritariamente de mamíferos marinos, reconociéndose pinnípedos y cetáceos (Tabla 3). Los pinnípedos fueron clasificados a nivel de familia

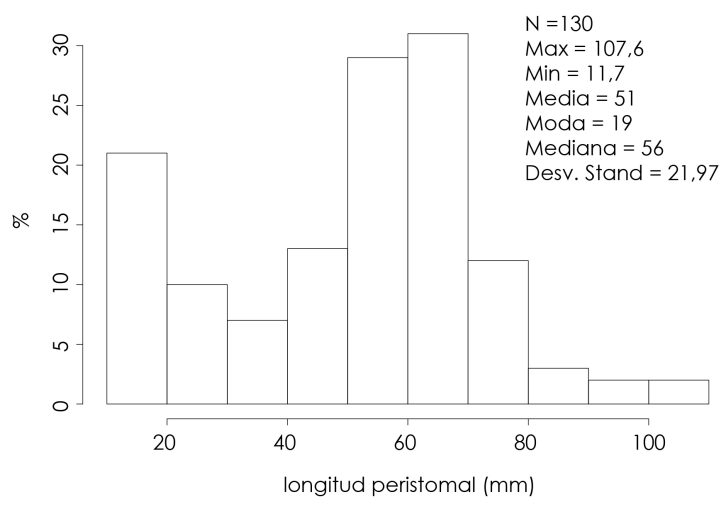

Figura 5. Estructura de talla de Concholepas concholepas. Las medidas indican la captura de especímenes pequeños (12-24 mm) y medianos (40-72 mm), en acuerdo a estudios arqueomalacológicos locales (Castelleti y Maltrain 2010; Olguín 2013, 2014; Olguín et al. 2015b).

Size structure of Concholepas concholepas. Measurements indicate the capture of small (12-24 mm) and medium (40-72 mm) specimens according to local archaeomalacological studies (Castelleti and Maltrain 2010; Olguín 2013, 2014; Olguín et al. 2015b). 
Tabla 3. Restos identificados de mamíferos, aves y peces en Caleta Bandurrias. Remains of mammals, birds, and fishes identified at Caleta Bandurrias.

\begin{tabular}{|c|c|c|c|c|c|c|c|c|}
\hline \multirow{2}{*}{ Taxa } & \multicolumn{6}{|c|}{ NISP } & \multicolumn{2}{|c|}{ MNI } \\
\hline & Estructura 1 & Cuadrícula 1 & $\begin{array}{c}\text { Perfil 6- } \\
\text { Columna } 1\end{array}$ & $\begin{array}{c}\text { Perfil 5- } \\
\text { Columna } 2\end{array}$ & Total & $\%$ & Total & $\%$ \\
\hline Otariidae & 60 & 151 & 10 & 3 & 224 & 64,37 & 14 & 48,28 \\
\hline Camelidae & 24 & 60 & 7 & 2 & 93 & 26,72 & 6 & 20,69 \\
\hline Delphinoidea & 1 & 1 & 11 & & 13 & 3,74 & 3 & 10,34 \\
\hline Cetacea & 8 & 5 & & & 13 & 3,74 & 2 & 6,90 \\
\hline Sigmodontinidae & 2 & 1 & & & 3 & 0,86 & 2 & 6,90 \\
\hline Canidae & 1 & 1 & & & 2 & 0,57 & 2 & 6,90 \\
\hline $\begin{array}{l}\text { NISP Total } \\
\text { mamíferos }\end{array}$ & 96 & 219 & 28 & 5 & 348 & 100 & 29 & 100 \\
\hline Mamíferos indet. & 437 & 389 & 344 & 60 & 1.230 & & & \\
\hline Total mamíferos & 533 & 608 & 372 & 65 & 1.578 & & & \\
\hline Laridae & & 7 & & & 7 & 30,43 & 1 & 12,50 \\
\hline Larus sp. & & 2 & & & 2 & 8,70 & 1 & 12,50 \\
\hline Phalacrocorax sp. & & 4 & & & 4 & 17,39 & 1 & 12,50 \\
\hline Pelecanus thagus & & 4 & & & 4 & 17,39 & 1 & 12,50 \\
\hline Spheniscus sp. & & 2 & & & 2 & 8,70 & 1 & 12,50 \\
\hline Procellariformes & & 1 & & & 1 & 4,35 & 1 & 12,50 \\
\hline Puffinus sp. & & 3 & & & 3 & 13,04 & 1 & 12,50 \\
\hline NISP Total aves & & 23 & & & 23 & 100 & 1 & 12,50 \\
\hline Aves indet. & 37 & 31 & 16 & 31 & 115 & & 8 & 100 \\
\hline Total aves & 37 & 54 & 16 & 31 & 138 & & & \\
\hline Trachurus murphyi & 62 & 254 & 104 & 36 & 456 & 81,28 & 104 & 78,20 \\
\hline Paralichthys microps & & 31 & & & 31 & 5,53 & 2 & 1,50 \\
\hline $\begin{array}{l}\text { Semicossyphus } \\
\text { darwini }\end{array}$ & 6 & 11 & 8 & 4 & 29 & 5,17 & 7 & 5,26 \\
\hline Chondrichthyes & 4 & & 4 & 1 & 9 & 1,60 & 3 & 2,26 \\
\hline Xiphias gladius & 5 & 3 & & & 8 & 1,43 & 2 & 1,50 \\
\hline Cilus gilberti & & 2 & 3 & 2 & 7 & 1,25 & 3 & 2,26 \\
\hline $\begin{array}{l}\text { Ethmidium } \\
\text { maculatum }\end{array}$ & 5 & & & & 5 & 0,89 & 1 & 0,75 \\
\hline Thyrsites atun & 2 & 2 & & & 4 & 0,71 & 2 & 1,50 \\
\hline Genypterus spp. & & 3 & & & 3 & 0,53 & 1 & 0,75 \\
\hline Graus nigra & 1 & & 1 & & 2 & 0,36 & 2 & 1,50 \\
\hline $\begin{array}{l}\text { Cheilodactylus } \\
\text { variegatus }\end{array}$ & & & 2 & & 2 & 0,36 & 1 & 0,75 \\
\hline $\begin{array}{l}\text { Aplodactylus } \\
\text { punctatus }\end{array}$ & 1 & & 1 & & 2 & 0,36 & 2 & 1,50 \\
\hline Isacia conceptionis & & 1 & & & 1 & 0,18 & 1 & 0,75 \\
\hline Prolatilus jugularis & & & 1 & & 1 & 0,18 & 1 & 0,75 \\
\hline Girella leavifrons & & 1 & & & 1 & 0,18 & 1 & 0,75 \\
\hline NISP Total peces & 86 & 308 & 124 & 43 & 561 & 100 & 133 & 100 \\
\hline Teleostei indet. & 204 & 281 & 373 & 162 & 1.020 & & & \\
\hline Total peces & 290 & 589 & 497 & 205 & 1.581 & & & \\
\hline Total & 860 & 1.251 & 885 & 301 & 3.297 & & 170 & \\
\hline
\end{tabular}

(Otariidae), correspondiendo al taxón con mayor representación. Se determinó un número mínimo de tres individuos: un individuo de edad adulta, un juvenil y un neonato. La presencia de neonatos, es indicativa del periodo de pariciones de las hembras, considerándose un intervalo máximo entre los meses de noviembre y febrero, en acuerdo a los patrones reproductivos de otáridos con distribución actual en la zona (Acevedo et al. 2003; Pavés et al. 2005; Sielfeld 1999). Las poblaciones del litoral continental de Chile, 
actualmente se distribuyen en parches en roqueríos costeros, islotes y playas de arena, constituyendo sus paraderos o colonias reproductivas (Sielfeld 1999). Los métodos locales para la captura de dichos mamíferos refieren al apaleo en tierra con garrotes y/o a la caza con arpón desde embarcaciones (Ballester 2017). Se sugiere la aplicación del último método, en correspondencia con el acceso a los islotes que confrontan la puntilla Bandurrias. La determinación anatómica de otáridos, por su parte, diagnostica la presencia de segmentos del esqueleto apendicular y axial, lo cual plantea el ingreso de presas completas al yacimiento (Tabla 4).

Respecto a los cetáceos, se identificaron mayoritariamente restos de Delphinidae. Los especímenes corresponden a fragmentos de cráneo, vértebras y un hueso tímpano-periótico posiblemente atribuible al género Lissodelphis (Kasuya 1973; Martínez 2008). El registro de cetáceos, aunque escaso, da luces del aprovechamiento de estos mamíferos en episodios de encallado y/o en el marco de incursiones en alta mar orientada a la caza de distintas especies oceánico-pelágicas (Aguayo-Lobo et al. 1998).

Los mamíferos terrestres, en tanto, se representan mayoritariamente por restos de camélidos y, en menor medida, por roedores caviomorfos y cánidos. Los restos de camélidos permitieron identificar la presencia de un individuo adulto y un juvenil de sexo indeterminado. El perfil anatómico de dicho taxa se muestra bastante incompleto, registrándose casi exclusivamente huesos del autopodio ${ }^{2}$ (Tabla 4). La especie endémica de la costa árida del norte de Chile es Lama guanicoe, la cual habita en bajas densidades en parches de vegetación localizados de los oasis de niebla de la Cordillera de la Costa durante todo el ciclo anual (González et al. 2006; Raedeke y Simonetti 1988). Dichas características en cuanto a su distribución y estructura poblacional, permiten sugerir una modalidad de explotación más específica de dicho taxa por parte de las poblaciones costeras.

\section{Variabilidad artefactual y organización tecnológica}

\section{Artefactos óseos}

Se constatan 15 ejemplares pulidos correspondientes a siete categorías morfofuncionales (Tabla 5; Figura 6). La categoría principal está definida por cuatro artefactos con borde redondeado y sección plana, caracteres que permiten asignarlos a chopes/desconchadores de morfología conocida (Berenguer 2009; Bird 1943; Standen y Arriaza 2016). Se registran, adicionalmente, puntas romas (compresores/retocadores), barbas de arpón y de anzuelo compuesto, una pesa, un objeto perforado, y tres artefactos de sección circular con extremo aguzado. Los atributos de estos últimos, sugieren una función punzante, más no se descarta
Tabla 4. MNE, MAU y \%MAU de Camelidae y Otariidae. MNE, MAU and \%MAU of Camelidae and Otariidae.

\begin{tabular}{|c|c|c|c|c|c|c|}
\hline \multirow{2}{*}{ Especímen } & \multicolumn{3}{|c|}{ Otariidae } & \multicolumn{3}{|c|}{ Camelidae } \\
\hline & MNE & MAU & $\% \mathrm{MAU}$ & MNE & MAU & $\% \mathrm{MAU}$ \\
\hline Cráneo & 2 & 2,00 & 66,67 & 1 & 1,00 & 22,22 \\
\hline Mandibula & 2 & 1,00 & 33,33 & 3 & 1,50 & 33,33 \\
\hline Axis & 3 & 3,00 & 100 & & & \\
\hline $\begin{array}{l}\text { Vértebras } \\
\text { cervicales }\end{array}$ & 2 & 0,40 & 13,33 & 2 & 0,40 & 8,89 \\
\hline $\begin{array}{l}\text { Vértebras } \\
\text { torácicas }\end{array}$ & 5 & 0,33 & 11,11 & 1 & 0,07 & 1,48 \\
\hline $\begin{array}{l}\text { Vértebras } \\
\text { lumbares }\end{array}$ & 4 & 1,00 & 33,33 & 1 & 0,20 & 4,44 \\
\hline Sacro & 3 & 1,00 & 33,33 & & & \\
\hline $\begin{array}{l}\text { Vértebras } \\
\text { caudales }\end{array}$ & 2 & 0,20 & 6,67 & & & \\
\hline Vértebras indet. & 4 & - & & & & \\
\hline Costillas & 18 & 0,60 & 20,00 & 4 & 0,17 & 3,70 \\
\hline Esternebra & 3 & 0,38 & 12,50 & & & \\
\hline Baculum & 1 & 1,00 & 33,33 & & & \\
\hline Escápula & 2 & 1,00 & 33,33 & 9 & 4,50 & 100 \\
\hline Húmero px & 4 & 2,00 & 66,67 & & & \\
\hline Húmero ds & 1 & 0,50 & 16,67 & & & \\
\hline Radio px & 2 & 1,00 & 33,33 & 2 & 1,00 & 22,22 \\
\hline Radio ds & 1 & 0,50 & 16,67 & & & \\
\hline Ulna px & 2 & 1,00 & 33,33 & & & \\
\hline Ulna ds & 1 & 0,50 & 16,67 & & & \\
\hline Carpos & 7 & 0,50 & 16,67 & 3 & 0,21 & 4,76 \\
\hline Metacarpo px & 3 & 0,30 & 10,00 & 1 & 0,50 & 11,11 \\
\hline Coxal & 3 & 1,50 & 50,00 & & & \\
\hline Fémur px & 2 & 1,00 & 33,33 & & & \\
\hline Fémur ds & 2 & 1,00 & 33,33 & & & \\
\hline Patella & 1 & 0,50 & 16,67 & 3 & 1,50 & 33,33 \\
\hline Tibia px & 3 & 1,50 & 50,00 & 2 & 1,00 & 22,22 \\
\hline Tibia ds & 1 & 0,50 & 16,67 & 1 & 0,50 & 11,11 \\
\hline Fíbula & 1 & 0,50 & 16,67 & & & \\
\hline Astrágalo & & & & 1 & 0,50 & 11,11 \\
\hline Calcáneo & 1 & 0,50 & 16,67 & & & \\
\hline Tarso & 1 & 0,10 & 3,33 & 1 & 0,1 & 2,22 \\
\hline Metatarso px & 3 & 0,30 & 10,00 & 3 & 1,50 & 33,33 \\
\hline Metatarso ds & 2 & 0,20 & 6,67 & & & \\
\hline 1 falange & & & & 4 & 0,50 & 11,11 \\
\hline 2 falange & & & & 4 & 0,50 & 11,11 \\
\hline 3 falange & & & & 1 & 0,13 & 2,78 \\
\hline Falanges & 13 & 0,23 & 7,74 & 4 & 0,17 & 3,70 \\
\hline Total NRD & 105 & & & 51 & & \\
\hline
\end{tabular}

su adscripción como barbas de potera (Labarca et al. 2017).

\section{Artefactos conquiológicos}

La muestra consta de seis artefactos pulidos sobre Choromytilus chorus, registrándose dos 


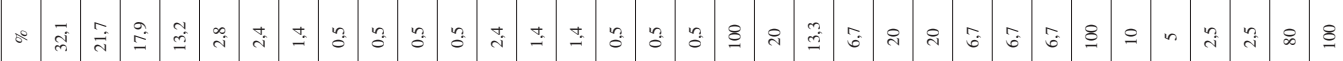

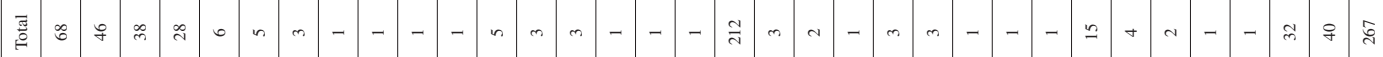
童喜

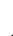


a.

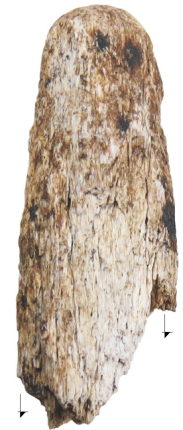

0

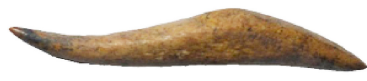

b.

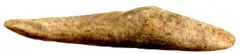

d.

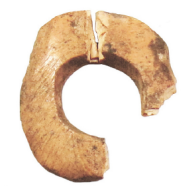

f. c.

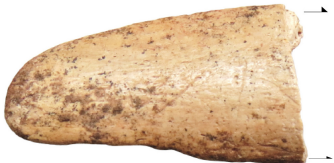

Figura 6. Artefactos óseos. a. pesa; b. barba de arpón; c. chope/desconchador; d. barba de arpón; e. compresor/retocador; f. objeto perforado; g. artefacto con extremo aguzado.

Bone artifacts. a. fishing weight. b. barb for harpoon forepieces; $c$. shellfish detachers; $d$. barb for harpoon forepieces; e. pressure flaker; f. perforated object; g. artifact with sharp end.

anzuelos con vástago y cuatro fragmentos de pesas cilíndricas, dos de los cuales corresponden a vástagos de anzuelos compuestos (Bird 1943) (Tabla 5;
Figura 7). La fragmentación de los artefactos óseos y conquiológicos indica el descarte de piezas al fin de su vida útil y en etapas de elaboración. En este

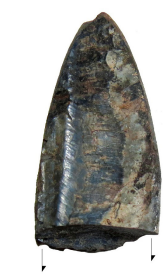

a.
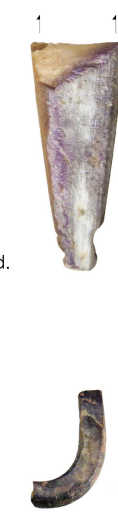

g. b.

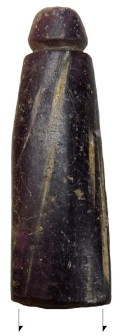

c.
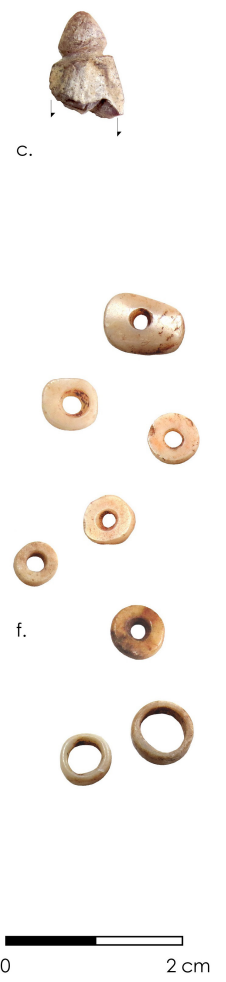

Figura 7. Artefactos conquiológicos. a. charnela pulida de Choromytilus chorus; b-e. pesas/vástagos de anzuelo compuesto de Choromytilus chorus; f. cuentas; g. anzuelo de Choromytilus chorus; g. fragmento pulido de valva de Choromytilus chorus.

Shell artifacts. a. polished internal edge of mussel shell; b-e.mussel shell fishing weights/shanks; f. shell beads g. mussel shell fishhook; h. polished fragment of mussel shell. 
sentido, se consignan dos fragmentos pulidos de valva de Choromytilus, correspondientes a una matriz de anzuelo y una preforma de pesa (Bird 1943; Flores et al. 2016). Además, se registraron 32 cuentas de matriz indeterminada, procedentes casi exclusivamente de la Estructura-1, sugiriendo su adherencia a un mismo colgante (Tabla 5). Los análisis morfométricos señalan la predominancia del tipo discoidal mediano $(\mathrm{N}=19) \mathrm{y}$, minoritariamente, de los tipos discoidal-pequeño $(\mathrm{N}=2)$ y rectangular $(\mathrm{N}=2)$ (cf. Soto et al. 2018).

\section{Artefactos líticos}

Se identificaron artefactos pulidos (2\%), pulidopiqueteados $(8 \%)$ y tallados (90\%) (Tabla 5). Los artefactos pulidos corresponden a categorías que comportan funciones análogas o complementarias al conjunto conquiológico. De esta manera, se registra un fragmento de pesa cilíndrica de andesita y un fragmento de "lima" de arenisca, vinculada a la manufactura de anzuelos de concha (Bird 1943; Durán 1985; Llagostera 1989; Silva y Bahamondes
1969). Por su parte, se constata una cuenta tubular lítica posiblemente relacionada al conjunto de cuentas de concha anteriormente descrito ${ }^{3}$ (Figura 8).

Los artefactos pulido-piqueteados constituyen piezas sin formatización, sobre guijarros graníticos y andesíticos con distribución local en fondos de quebradas, desembocaduras o playas de guijarros (Castelleti 2007; Galarce y Santander 2013; Guendon 2009; Silva y Bahamondes 1969). Estos se asocian predominantemente a actividades de procesamiento de óxidos de hierro, en los que se incluyen sobadores/ aplicadores, manos de moler y soportes de molienda; todos ellos con rastros de dicho colorante (Figura 8). En menor medida se reconocieron artefactos para la producción lítica, compuestos por un percutor y un yunque/afilador.

Los artefactos tallados se confeccionaron principalmente sobre materias primas silíceas (Tabla 5). La categoría más frecuente corresponde a lascas con retoque marginal, las cuales refieren a una vasta gama de actividades posibles (Keeley 1982). La segunda categoría más representada son los cuchillos, siendo
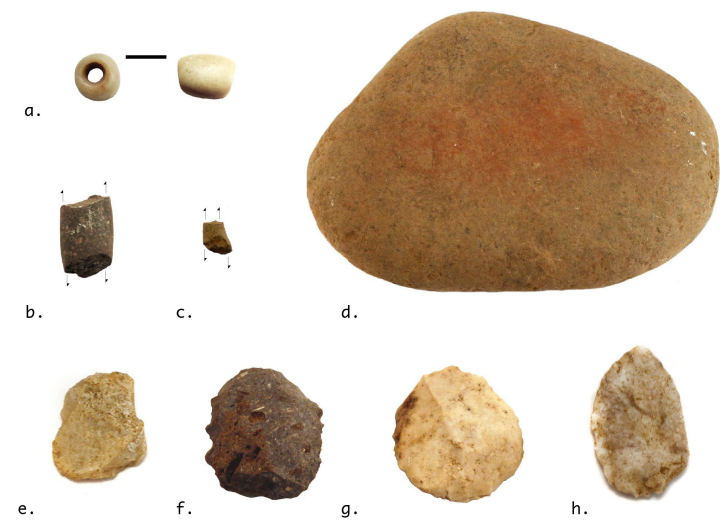

d.

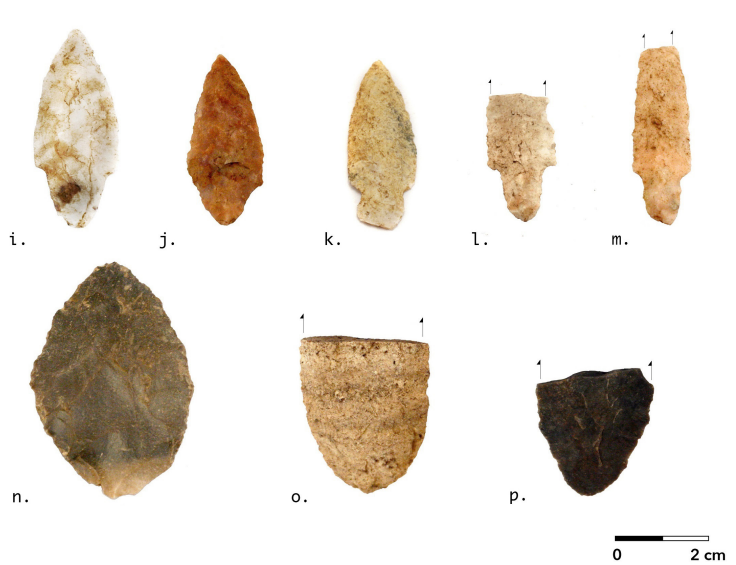

Figura 8. Instrumentos líticos. a. cuenta; b. pesa; c. fragmento de "lima" de arenisca; d. guijarro con pigmento; e-h. lascas retocadas; i-m. puntas de proyectil; $n$-p. cuchillos bifaciales.

Lithic tools. $a$. bead; $b$. weight fragment; c. file fragment; $d$. pigmented pebble; $e$-h. flakes artifacts; i-m. projectile points; $n$-p. bifacial knives. 
recurrentes los ejemplares bifaciales. Destacan algunas piezas de morfología foliácea con acabado adelgazamiento bifacial y fragmentos con características compatibles (Figura 8). Igualmente, se consignan abundantes puntas de proyectil, en su mayoría pedunculadas. Estas pudieron funcionar como cabezales de dardos, lanzas y/o arpones, sin excluir su uso como bifaces enmangados. Algunos ejemplares completos o con remanentes mesoproximales $(\mathrm{N}=7)$, señalan tendencias hacia limbos lanceolados con pedúnculos convergentes o paralelos (Figura 8). De manera complementaria, se observa una alta proporción de preformas descartadas, dando cuenta de la elaboración de cabezales líticos en el yacimiento. En términos generales, el conjunto bifacial presenta un alto índice de fractura y reavivado de filos, sugiriendo el descarte de dichos ítems debido a la prescripción de su vida útil (Andrefsky 1994).

En tanto, el conjunto de derivados de talla $(\mathrm{N}=4.419)$ da cuenta de una intensiva actividad productiva en el asentamiento, en donde la alta proporción de derivados a instrumentos indica una manufactura orientada a la elaboración y utilización in situ del instrumental obtenido (Andrefsky 1998). El análisis permitió constatar un notorio predominio de rocas silíceas $(\mathrm{NMD}=2.326 ; 94 \%)$, identificándose tobas silicificadas, chert, calcedonias y jaspes de diversos colores y texturas (Luedtke 1992). A la fecha se reconocen tres fuentes potenciales de rocas silíceas/silicificadas en el desierto interior de TaltalPaposo, a 45-80 km de distancia (Borie et al. 2017, 2018). En relación a dicha variable, se observa una alta frecuencia de derivados carentes de cobertura cortical (97\%), indicando la incorporación de matrices previamente trabajadas al sitio. Asimismo, se registran un predominio de plataformas facetadas $(43 \%)$, siendo minoritarias las morfologías planas (24\%), rebajadas $(23 \%)$ y puntiformes (10\%). Por su parte, la distribución de tamaños demuestra una tendencia hacia derivados pequeños (Figura 9). Dichos atributos en conjunto, apuntan a actividades de reducción bifacial y de regularización y/o mantenimiento de filos y/o puntas (Andrefsky 1998).

Las rocas no silíceas están representadas mayoritariamente por andesitas afaníticas de disponibilidad local (NMD=66) (Guendon 2009). Este grupo petrográfico se compone por derivados sin corteza (88\%), con talones planos (57\%), corticales (36\%) y diedros (7\%); registrando tamaños superiores respecto a las materias silíceas (Figura 9). Lo anterior indica etapas iniciales de reducción, orientadas a la obtención de formas base y al desbaste marginal. En menor medida se identifican derivados de cuarzo (NMD=16), con talones planos (54\%), rebajados (16\%), diedros (15\%) y corticales (15\%), aludiendo a su obtención local en vetas del batolito costero (Gaete et al. 2003; Guendon 2009). Finalmente, el registro de dos desechos de retoque de obsidiana sugiere la incorporación de instrumentos terminados sobre dicha materia prima. Vale señalar, que la disponibilidad de obsidiana es exclusiva a pisos altoandinos y quebradas prepuneñas de laregión(DeSouzaetal. 2002; Seelenfreund et al. 2010), lo cual aporta nueva evidencia sobre las redes de circulación costa-interior funcionando durante este momento de la secuencia prehispánica (Ballester y Gallardo 2011; De Souza et al. 2010; Núñez et al. 1974; Núñez y Perlès 2018; Soto et al. 2018).

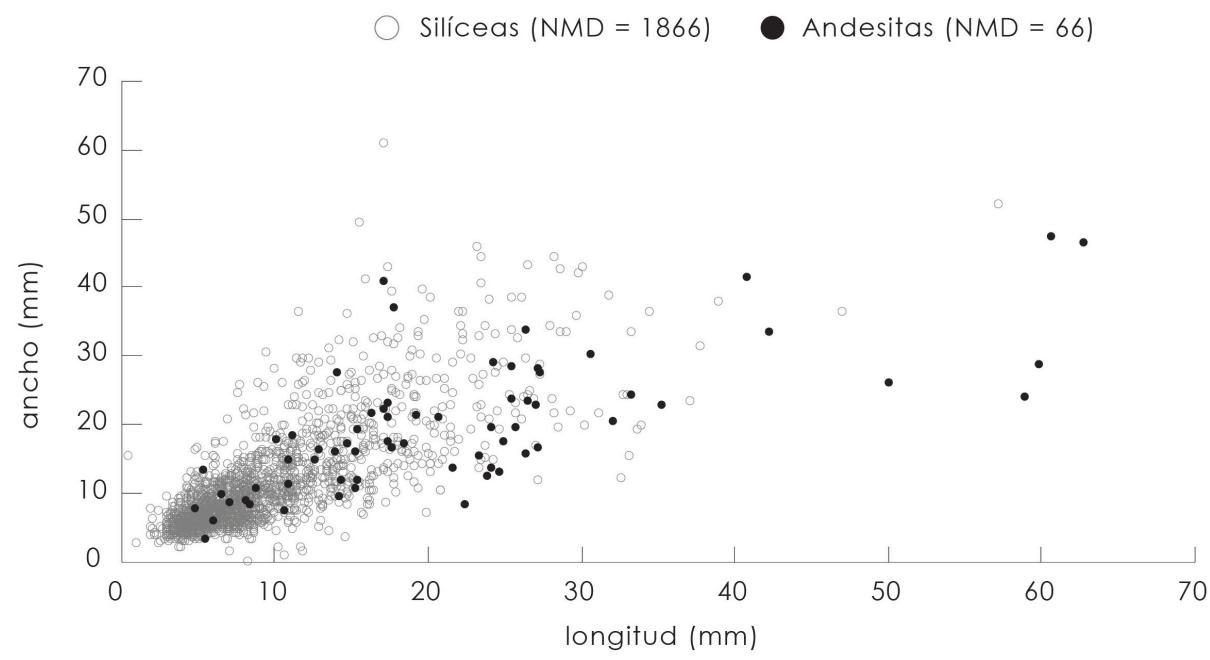

Figura 9. Distribución de tamaños de derivados líticos.

Flake-size distribution of lithic derivatives. 


\section{Discusión}

La ubicación del sitio Caleta Bandurrias presenta condiciones favorables para su habitabilidad y articulación de circuitos de desplazamiento longitudinales y latitudinales durante la(s) fase(s) estacionaria(s) de su ocupación. Se constata la presencia de tres aguadas, loberas, colonias de aves y una caleta protegida, con óptimas condiciones de disponibilidad y acceso a los recursos litorales y marítimos de fundamental interés para los grupos cazadores recolectores y pescadores de la zona. Asimismo, la quebrada Bandurrias constituye una ruta natural directa hacia el desierto interior, favoreciendo el acceso a materias primas y/o recursos distantes o, concretamente, para el encuentro con otras comunidades de la región (Ballester y Crisóstomo 2017; Ballester y Gallardo 2011; Borie et al. 2018; Soto et al. 2018). Estas variables de emplazamiento concuerdan con aquellas ampliamente documentadas en diferentes yacimientos del Holoceno Medio del litoral arreico (Andrade y Salazar 2011; Ballester y Gallardo 2011; Ballester et al. 2017, 2018; Bustos 1974; Castelleti 2007; Llagostera 1989, 2005; Núñez 1984; Núñez y Varela 1967-68; Salazar et al. 2015; entre otros).

En relación a los conchales asociados a los conjuntos arquitectónicos, destaca su moderada potencia y baja complejidad estratigráfica y resolución, cualidades que parecen ser propias de los basurales de yacimientos con arquitectura del Arcaico Tardío y que contrastan con los densos conchales monticulares de data previa (Bird 1946; Castelleti 2007; Durán 1985; Llagostera 1989; 2013; Mostny 1964; Núñez 1971; Núñez et al. 1974; Salazar et al. 2015). Lo anterior problematiza, en parte, la intensidad y estabilidad de la ocupación del yacimiento, en lo respectivo a las tasas de depositación esperables para un asentamiento permanente o semipermanente (Arnold 1996; Chatters 1987; Kelly 1992; Stein et al. 2003). En esta misma línea, y en acuerdo a planteamientos previamente realizados por Zlatar $(1983,1987)$, vale considerar si efectivamente los depósitos-conchales de los sitios con arquitectura Caleta Huelén-42 de la costa arreica corresponden a acumulaciones producidas durante ocupaciones continuas de carácter semipermanente, o si más bien derivan de la frecuentación periódica y/o de corta duración de las comunidades a los asentamientos respectivos (cf. Aldeias y Bicho 2016; Stein et al. 2003).

De momento, el amplio espectro de recursos identificados en los depósitos de Caleta Bandurrias da cuenta de su obtención en actividades de caza, pesca y recolección en distintos ambientes costeros y del interior, mediante diferentes equipos y tecnologías. Las labores realizadas en el borde costero se fundaron en la recolección de diversos invertebrados, siendo ubicuos los gastrópodos, poliplacóforos y equinodermos de alta biomasa. Los peces de roca, aunque minoritarios, posiblemente fueron capturados individualmente; en tanto, los peces pelágicos de mayor representatividad pudieron ser apresados durante sus incursiones masivas hacia la costa, generalmente referidas a periodos estivales (Béarez et al. 2016; Capdeville 1921a; Contreras 2011; Rebolledo et al. 2016;). Los resultados indican, igualmente, incursiones fuera del litoral, ya fuese a los islotes asociados a la puntilla, como también hacia aguas oceánicas para la caza de mamíferos marinos y de peces pelágicos, respectivamente. Tales actividades sugieren el uso de embarcaciones, a la vez que una imbricada organización para la captura y traslado de dichas presas hasta el yacimiento (Ballester 2018; Ballester et al. 2017; Béarez et al. 2016; Castro et al. 2016; Olguín et al. 2014, 2015a; Rebolledo et al. 2016). Por su parte, la representación de valvas y segmentos esqueletarios de alto y bajo valor económico de prácticamente la totalidad de taxa costerosmarítimos representados, sugiere que estos se estarían explotando, transportando, procesando y consumiendo in situ, expectativas propias de un campamento residencial (Chatters 1987). Dichas prácticas fueron factibles de realizar en distintos loci de la puntilla, tanto en la caleta misma, como en los roqueríos, loberas y playas aledañas, evidenciándose un estrecho vínculo del asentamiento con la estructura ambiental inmediata (Chatters 1987). De esta manera, el entorno costero e inshore de Caleta Bandurrias constituyeron locaciones extractivas de acceso diario, definiendo el radio forrajero de las poblaciones durante su estadía en el yacimiento ${ }^{4}$ (7-10 km; cf. Binford 1983, 2001; Kelly 1995).

La explotación minoritaria del guanaco, por su parte, da cuenta de circuitos de forrajeo dirigidos a espacios propios del ecosistema de lomas, los cuales bien pudieron corresponder a cimas, laderas, y quebradas del tramo inferior de la Cordillera de la Costa, como también a cotas más alejadas de la Plataforma Litoral (Ballester y Gallardo 2011; Ballester et al. 2017, 2018; Castelleti 2007; Núñez 1984; Salazar et al. 2015). En función de la distribución, tamaño y perfil anatómico de dicho taxa, se plantea el ingreso de las presas en estado incompleto al sitio, ya fuese en el marco de conductas de transporte selectivo durante capturas ocasionales (cf. hunting trips; Binford 1980) o de la explotación dirigida de sus restos óseos como materia prima (Olguín et al. 2015a). Vale mencionar las tendencias de aprovechamiento de invertebrados, peces y mamíferos observadas en el conjunto arqueozoológico de Caleta marinos del Holoceno Medio en Taltal (Béarez et al. 2016; Castelleti 2007; Olguín 2013, 2014; Olguín et al. 2015b; Rebolledo et al. 2016 Salazar et al. 2015).

En una particular conjunción de ambientes, materias y tecnologías, las comunidades que ocuparon el sitio Caleta Bandurrias diseñaron y conformaron 
distintos equipos tecnológicos para su convivencia con el medio marino durante su(s) estadía(s) en el sitio. La gama más diagnóstica de útiles vinculados a la pesca, caza y recolección costero-marítima corresponde a anzuelos, pesas, barbas de retención y chopes/ desconchadores. Dichos artefactos fueron elaborados, principalmente, sobre valvas de Choromytilus chorus y huesos de camélido, demostrándose la reconocida importancia tecnológica de dichos taxa (Ballester 2018; Bird 1943; Boisset et al. 1969; Durán 1985; Flores et al. 2016; Labarca et al. 2017; Llagostera 1989, 2005; Olguín et al. 2015a; Silva y Bahamondes 1969). Los artefactos líticos, por su parte, evidencian una multiplicidad de actividades desarrolladas en el sitio, identificándose el uso de materias primas de disponibilidad local y no local. El aprovechamiento de rocas costeras consistió en la utilización de bloques o guijarros sin modificación, tanto para actividades productivas como para la conformación de las unidades arquitectónicas. Asimismo, constituyeron materias primas idóneas para la elaboración de artefactos pulidos y tallados. Las rocas silíceas de la pampa desértica (40 $\mathrm{km}$ al interior), conforman el conjunto de artefactos tallados más abundante, destacando los artefactos sobre lascas, instrumentos generalizados, versátiles y flexibles que pudieron servir para una vasta gama de actividades (Chatters 1987; Nelson 1991). Igualmente, son frecuentes los cabezales y cuchillos, posiblemente asociados a las actividades especializadas de caza y procesamiento de las grandes presas representadas en el conjunto ecofactual (Ballester et al. 2014b, 2017, 2018; Bird 1943; Boisset et al. 1969; Castelleti 2007; Castro et al. 2016 Galarce y Santander 2013; García-Albarido 2012; Latcham 1939; Núñez 1984).

En términos de la producción lítica, se constata que la proporción de derivados de talla e instrumentos se asocia a la manufactura y utilización in situ de los artefactos fabricados, vinculada a una lógica ocupacional de carácter residencial (Galarce y Santander 2013). En esta misma línea, las características tecnológicas de los derivados se corresponden con las categorías instrumentales más recurrentes, evidenciándose una actividad de talla orientada a la producción de artefactos bifaciales y a la preparación y/o mantenimiento de instrumentos. Dichas particularidades sugieren un aprovechamiento íntegro del recurso silíceo en proyección de su uso futuro, estrategia tecnológica adecuada a las condiciones de disponibilidad en áreas fuente no mediatas (Andrefsky 1994; Nelson 1991). Desde una perspectiva declaradamente no dicotómica, las prácticas forrajeras documentadas en Caleta Bandurrias se habrían complementado con el despliegue de movimientos latitudinales a larga distancia hacia tramos altos de las Cordillera de la Costa, la amplitud de la Depresión Intermedia y, eventualmente, hacia la
Cordillera de los Andes, conformando el radio logístico y/o extendido de los grupos litorales (sensu Binford 1983). Lo anterior es coherente con las propuestas de aprovisionamiento lítico costero de la región (Blanco et al. 2010; 2018; Castelleti 2007; Figueroa 2007; Urrejola y Orellana 1999, 2000), aun cuando los accesos de larga distancia bien pudieron tener motivaciones más complejas que exclusivamente el abastecimiento de materias primas (Ballester y Crisóstomo 2017; Ballester y Gallardo 2011; Borie et al. 2018; Pimentel et al. 2011; 2017; Pimentel y Ugarte 2017).

De esta manera, las características de emplazamiento, la presencia de al menos 27 estructuras arquitectónicas inmuebles, evidencia(s) funeraria(s) y de la estructura y composición del registro arqueofaunístico y artefactual, permiten postular que el sitio Caleta Bandurrias fue utilizado como una base residencial por grupos cazadores-recolectores-pescadores de la costa arreica al menos durante el rango entre los ca. 5800 - 4600 años cal. AP. En dicho espacio se habrían organizado y desarrollado la mayoría de las actividades domésticas y productivas, conformando el centro del radio de forrajeo y abastecimiento, así como también el área de co-residencia y socialización (Binford 1980 , 1983, 2001). Cabe señalar que los indicadores aquí discutidos respaldan igualmente la posibilidad de que el sitio haya funcionado como una locación de eventos cíclicos de agregación promovidos por factores sociales, rituales y/o económicos particulares (Guráieb 2011). En efecto, las agregaciones multibanda suelen ocurrir en puntos de intersección de los circuitos de desplazamiento y en lugares con abundantes recursos (Fitzhugh et al. 2011). Los sitios de agregación social:

son en principio, bases residenciales localizadas en ambientes especialmente favorables, con un buen acceso a los recursos de subsistencia y sin impactos humanos negativos de importancia sobre el paisaje que pudieran hacer no deseable la instalación humana a lo largo del tiempo (Guráieb 2001:376).

En tanto constituyen el campamento de unidades sociales agregadas, los lugares de estas características conforman un complejo composite de actividades múltiples que incluyen la elaboración de artefactos, y la preparación y consumo de diversos tipos de recursos (Binford 2001, 2006; Hitchcock y Ebert 2011). Lo anterior insta a continuar evaluando los indicadores que permitan dar cuenta de la variabilidad de asentamientos que caracterizan la creciente complejización del uso del espacio costero durante el Arcaico Tardío (Ballester y Gallardo 2011; Castelleti 2007; Power 2017; Salazar et al. 2015). 
A la fecha, se constatan al menos 16 yacimientos con conjuntos arquitectónicos asignables al rango cronológico de estudio entre la desembocadura del Loa y Punta Flamenco: Caleta Huelén-42 (Núñez 1971; Núñez y Santoro 2011; Núñez et al. 1975; Zlatar 1983, 1987), Copaca-1 (Andrade et al. 2016; Castro et al. 2012, 2016; Olguín et al. 2015a), Cobija-13 y S1 (Bittman 1984; Bittman y Munizaga 1984), Punta Guasilla-1 (Bittman y Munizaga 1984; Montenegro 1981), Aguada Gualaguala-04 (Ballester y Clarot 2014; Ballester et al. 2017, 2018), Chacaya-2 (Ballester et al. 2014a; Bustos 1974), Los Canastos-3 (Ballester et al. 2014b; Cruz y Llagostera 2011; Llagostera 2005, 2013), Zapatero (Salazar et al. 2015); Punta Negra-1a (Contreras et al. 2011); Punta Grande (Durán 1981, 1985); Caleta Bandurrias, Punta Morada, Morro Colorado, La Puntilla Sur (Bird 1943; Capdeville 1921b, 1928; Castelleti 2007; Mostny 1964; Núñez 1984; Salazar et al. 2015), Los Bronces-1 (Contreras et al. 2007, 2008; Llagostera y Llagostera 2010) y SM-26 (Salazar et al. 2015) (Figura 1)5. La existencia de campamentos sin arquitectura contemporáneos a los sitios mencionados, tales como Abtao-1 (Boisset et al. 1969), 228/230 (Castelleti 2007; Galarce y Santander 2013), Paso Malo Arcaico (Castelleti 2007; Salazar et al. 2015) y, posiblemente, Caramucho-3 (Olmos y Sanhueza 1984; Sanhueza 1980) y Cáñamo-1 (Núñez y Moraga 1977), demuestra distintas modalidades residenciales funcionando paralela y/o complementariamente a los campamentos con arquitectura (Ballester et al. 2017; Castelleti 2007; Power 2015, 2017; Salazar et al. 2015). Los datos del estudio intrasitio de Caleta Bandurrias y de los restantes sitios con ocupación residencial del periodo, permiten plantear el desarrollo de circuitos de movilidad fundamentados en el establecimiento y traslado de campamentos en torno a los recursos de subsistencia primaria en el tramo costero (Binford 1980; Kelly 1983, 1995) (Figura 10). En este sentido, las características de abundancia, disponibilidad, predictibilidad y baja estacionalidad de los recursos marinos (Yesner 1980) y los afloramientos hídricos localizados del litoral desértico (Kelly 1995; Taylor 1964), habrían consentido una movilidad residencial en donde las poblaciones organizarían sus espacios de vida en torno a dichos recursos, generando una cobertura completa de la franja litoral a partir de distintos campamentos residenciales autosuficientes y sus consecuentes loci extractivos ${ }^{6}$ (Binford 1980). Nuestro nivel de resolución temporal no permite por el momento sugerir que la totalidad de bases residenciales estén siendo ocupadas sincrónicamente, ni tampoco que estas constituyan campamentos estacionales dentro del circuito de movilidad residencial de una o múltiples unidades sociales, más dicha tarea deberá orientar trabajos futuros.

Con todo, la estrategia de movilidad aquí planteada difiere de recientes propuestas que abogan implícitamente por una movilidad de tipo logística operando para este momento de la secuencia prehispánica en la costa de Atacama (cf. Ballester y Gallardo 2011; Ballester et al. 2014b, 2014a; 2017, 2018). Específicamente, disentimos en lo referido al despliegue de múltiples campamentos logísticos en el eje litoral, puesto que dichas categorías funcionales corresponden a expectativas derivadas del modelo etnohistórico y están fundamentadas, exclusivamente, en base a dos yacimientos a lo largo del litoral arreico (Ballester y Gallardo 2011). Estos corresponden a los sitios 228/230 y Poza Bahamondes-4, los cuales sin embargo revisten distintos aspectos que problematizan su adscripción actual como campamentos logísticos (Ballester et al. 2017). De esta forma, el sitio 228/230 documenta prácticamente los mismos indicadores utilizados en la literatura reciente para adjudicar una función residencial a los sitios arquitecturizados, evidenciando densos depósitos de conchal, redundancia ocupacional, diversidad en la composición eco y artefactual, una intensiva producción lítica, áreas de actividad diferenciadas, e inclusive, un contexto funerario (Castelleti 2007; Castelleti y Maltrain 2010; Galarce y Santander 2013). Efectivamente, investigaciones actuales postulan un carácter residencial para el primer evento ocupacional del sitio y un uso más específico durante los episodios de re-ocupación (Galarce y Santander 2013). Por su parte, Poza Bahamondes-4 revela una modalidad ocupacional de moderada intensidad, caracterizada por una baja cantidad de restos de producción lítica y de fauna de consumo, interpretada como un "área de tareas específicas" articulada en torno a Morro Colorado y Punta Morada (Castelleti 2007:45). En ese sentido, y adhiriendo a la interpretación original del yacimiento, Poza Bahamondes-4 bien podría constituir uno de los diversos loci forrajeros de extracción y procesamiento diario, en especial considerando su ubicación a no más de 3 y $0,5 \mathrm{~km}$ respecto a los núcleos residenciales mencionados ${ }^{7}$. Todo lo anterior, sumado al cuantioso registro de bases residenciales y a la prácticamente nula cantidad de campamentos logísticos a nivel regional, denotan una espacialidad distintiva de una estrategia de movilidad de tipo residencial (Binford 1980, 2001; Chatters 1987; Kelly 1983, 1995).

Si bien por ahora no es posible precisar las motivaciones que subyacen a los movimientos residenciales periódicos planteados para la costa árida en este periodo, en especial considerando el carácter multidimensional que estas revelan en el registro etnográfico (Binford 2001; Kelly 1995; Politis 2006), algunas hipótesis podrían tener que ver con el impacto de la explotación humana en las estructuras poblacionales de los recursos costero-marítimos, sobre todo de ambiente litoral (cf. Olguín et al. 2015b), las diferencias estacionales en la abundancia relativa de presas de importancia económica (i.e. otáridos y/o peces neritopelágicos) (cf. Béarez et al. 2016; Rebolledo 


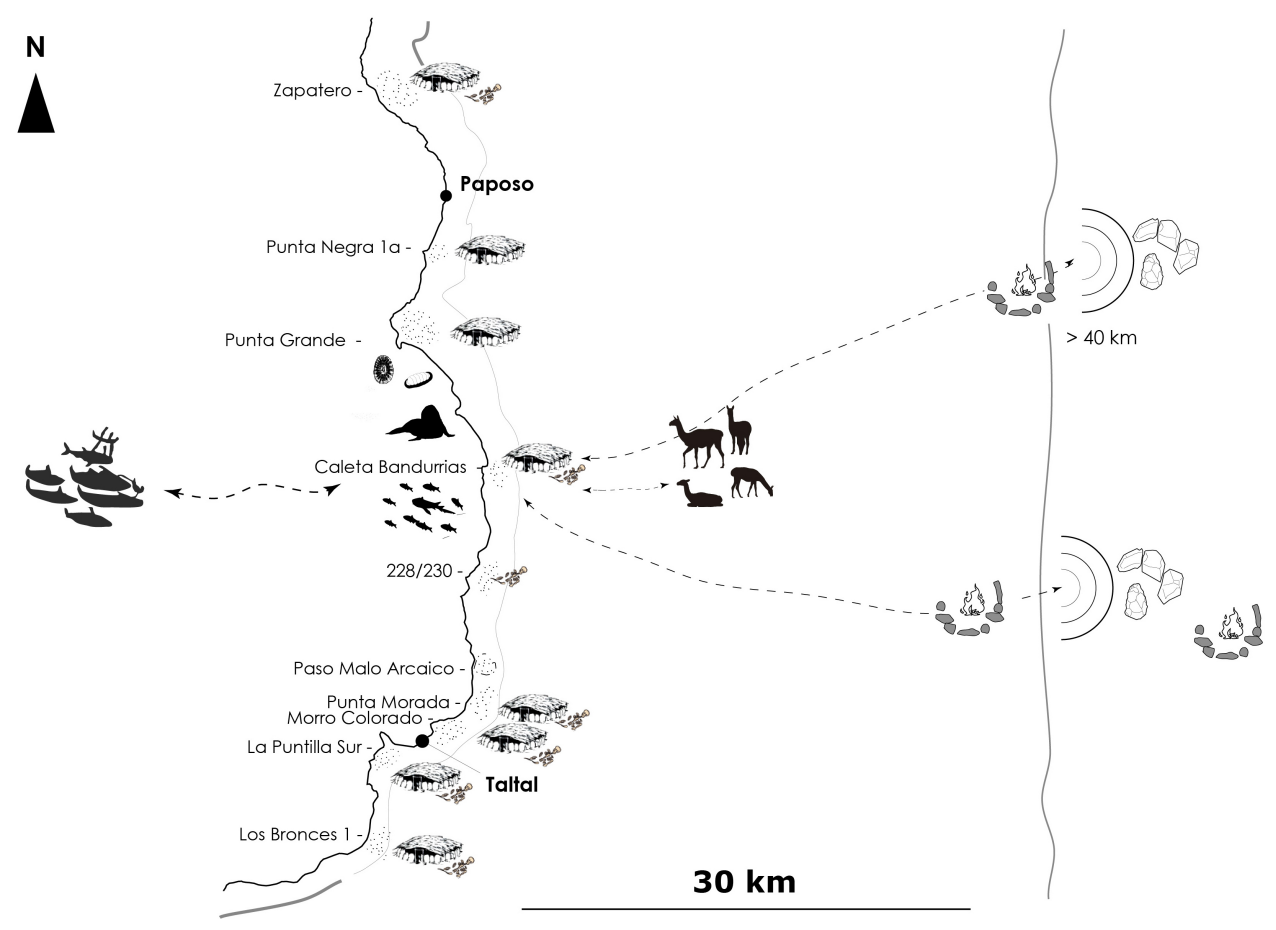

Figura 10. Modelo de la dinámica de asentamiento costero durante los ca. 5700-4000 cal. AP en el tramo Taltal-Paposo.

Model of coastal settlement dynamics between ca. 5700-4000 cal. BP on the Taltal-Paposo stretch.

et al. 2016), los ciclos de descarga y recarga de las aguadas costeras (cf. Herrera y Custodio 2014; Herrera et al. 2018), la eventual territorialidad de las mismas (Llagostera 1989, 2013), o bien con los requerimientos de reproducción social propios de estas poblaciones (Gamble 1983; Kelly 1995; Lazzari 2005; Macdonald y Hewlett 1999; MacEarchern 1994; Whallon 2006; Whallon et al. 2011), entre otros aspectos de su organización (Wiessner 1982, 1983; Wobst 1974, 2006). Más allá de cerrar esta problemática, resulta preciso la re-evaluación de las modalidades de ocupación intrasitio e intersitio a la luz de los nuevos datos derivados de la creciente investigación de la zona.

Un punto adicional que creemos necesario seguir profundizando dice relación con la permanencia en los campamentos y la frecuencia de la movilidad (sensu Chatters 1987). Actualmente contamos con el rango total de uso y algunos indicadores de estacionalidadque señalan al menos una ocupación estival para Caleta Bandurrias, más no hemos logrado una resolución temporal adecuada para profundizar en la(s) modalidad(es) de ocupación específica(s) y su(s) tiempo(s) de uso efectivo(s) (sensu Kelly 1995). Los límites de nuestro propio trabajo, invitan a ponderar de manera crítica las propuestas que han centrado la interpretación de los yacimientos con arquitectura bajo la idea de una ocupación permanente o semipermanente (Bittman 1984; Ballester y Gallardo 2011; Ballester et al. 2014b, 2014a, 2017, 2018; Castelleti 2007; Castro et al. 2016, Núñez et al. 1974; Salazar et al. 2015; entre otros). Si bien la arquitectura en piedra y patrones fúnebres formales han sido considerados generalmente como indicadores indirectos de una mayor estabilidad ocupacional en grupos cazadores recolectores (Binford 1990; Diehl 1992), estos no constituyen evidencias unívocas para sustentar dicha interpretación (Chatters 1987; Kelly 1992; Kelly et al. 2006; Marshall 2006; Monks 1981; Smith 2003). En efecto, las bajas tasas de depositación de los conchales asociados a estos yacimientos pueden ser igualmente entendidas como episodios de cohabitación o agregación social de carácter esporádico y/o estacional a lo largo del ciclo anual (Binford 2001). Adherimos, de esta forma, al uso residencial de los campamentos y al reconocido valor simbólico de la arquitectura residencial funeraria de la costa de Atacama, en términos de la consolidación de vínculos de ancestralidad con territorios específicos (Ballester et al. 2017, 2018; Núñez y Santoro 2011; Salazar et al. 2015). Dichas manifestaciones permiten plantear una lógica de largo plazo en relación al uso -si no 
continuo- al menos persistente y proyectado a futuro de dichos espacios, en el marco de los ciclos y circuitos de movilidad residencial de las comunidades litorales (Boyd 2006; Schlanger 1992; Smith 2003; Wandsnider 1992).

\section{Conclusión}

Los resultados obtenidos del estudio intrasitio de Caleta Bandurrias permiten asignar su uso residencial, funerario y/o de agregación social en el marco del asentamiento litoral de la costa arreica en el rango de los ca. 5800-4600 cal AP, entendiendo dichas modalidades de ocupación no necesariamente como categorías funcionales dicotómicas, sino como parte del complejo y diverso entramado de lugares que conforman el espacio de vida de las comunidades cazadoras-recolectoraspescadoras de Atacama (Maher y Conkey 2019). Desde los antecedentes disponibles, se establece que Caleta Bandurrias constituyó un campamento autosuficiente, centro del radio forrajero, de las actividades productivas y de la vida social de agregados sociales co-residentes, al menos por un(os) periodo(s) establecido(s) (Binford 2001). La variabilidad interna de los conjuntos eco y artefactuales señalan la concatenación de distintos rangos espaciales con respectivos loci de tareas desplegados en el medio litoral-marítimo y en los ecosistemas de lomas de la Cordillera de la Costa, complementados por movimientos logísticos dirigidos a la Depresión Intermedia y/o a las tierras altas de la región.

La adscripción funcional de Caleta Bandurrias si bien coincide con las interpretaciones de índole residencial de yacimientos coetáneos con arquitectura de la costa arreica, difiere con algunos aspectos más específicos de dichas investigaciones, como son las estrategias de movilidad y el carácter permanente de los asentamientos. Al ponderar los resultados de Caleta Bandurrias en el marco del patrón de asentamiento regional, se constata la existencia de cuantiosos campamentos residenciales y únicamente dos -empero, discutibles- campamentos logísticos referidos a lo largo de $800 \mathrm{~km}$ de extensión de la costa arreica. Lo anterior sugiere una dinámica de ocupación en base a una estrategia de movilidad residencial a lo largo del litoral, la cual sería promovida por la sustancial biomasa costero- marítima y los afloramientos hídricos localizados. Como componente estructurante del asentamiento costero, se consideran adicionalmente los conjuntos arquitectónicofunerarios que permitieron fundar, reclamar y disponer territorios específicos a determinadas unidades sociales en el marco de sus circuitos estables de movilidad residencial. De esta manera, la costa de Atacama arquitecturizada, se interpreta en la dinámica de un espacio persistente que invitó a su frecuentación y a la proyección de su uso en el largo plazo (Schlanger 1992).

Dada la complejidad intrínseca del estudio de conchales arqueológicos, la baja resolución de los depósitos de Caleta Bandurrias no permite de momento precisar su temporalidad y modalidad de uso específica, en lo respectivo a la residencia anual continua o a la redundancia ocupacional cíclica del yacimiento (Stein et al. 2003). Dicha reserva interpretativa -enfatizamos-, debiese ser aplicada a la totalidad de asentamientos con estas características de la costa del Desierto de Atacama. La evidencia arquitectónica-funeraria, de esta manera, no debe ser interpretada expresamente como la manifestación material de la vida sedentaria, pues dicha inferencia precisa de una contrastación con líneas de evidencia independientes (cf.; Aldeias y Bicho 2016; Andrus 2011; Burchell et al. 2013; Marshall 2006; Monks 1981; Stein et al. 2003), y su correspondiente evaluación con los procesos de transformación del sistema de asentamiento desde una perspectiva histórica de larga data (Arnold 1996).

Agradecimientos: Investigación financiada a través de los proyectos FONDECYT 1080666 y FONDECYT 1151203. Se agradece al equipo de investigación, especialmente a Laura Olguín, Victoria Castro, Pedro Andrade y Guido Gutiérrez por su apoyo en las actividades de terreno. A Camila Palma, Camila Opazo, Javiera Bastías, Catalina Soto, Boris Santander y Luca Sitzia por su apoyo en laboratorio y gabinete. Reconocemos con especial énfasis a Donald Jackson $\dagger$ por guiar la tesis que antecede este trabajo y por su ayuda en la comprensión de la historia ocupacional de la costa de Taltal y Paposo. Finalmente, se agradece a los evaluadores anónimos por sus observaciones y comentarios que contribuyeron a mejorar el manuscrito.

\section{Referencias Citadas}

Acevedo, J., A. Aguayo-Lobo y W. Sielfeld 2003. Eventos reproductivos del león marino común, Otaria flavescens (Shaw 1800), en el norte de Chile (Pacífico suroriental). Revista de Biología Marina y Oceanografía 38:69-75.

Aguayo-Lobo, A., D. Torres y J. Acevedo 1998. Los Mamíferos Marinos de Chile: Cetacea. Serie Científica INACH 48:19-159.
Aldeias, V. y N. Bicho 2016. Embedded behavior: human activities and the construction of the Mesolithic shellmound of Cabeço da Amoreira, Muge, Portugal. Geoarchaeology: An International Journal 31 (6):1-20.

Andrade, P. y D. Salazar 2011. Revisitando Morro Colorado: comparaciones y propuestas preliminares en torno a un conchal arcaico en las costas de Taltal. Taltalia 4:63-83. 
Andrade, P., V. Castro y C. Aldunate 2016. Reconstrucción del modo de vida de individuos del arcaico de la costa arreica del norte de Chile: una aproximación bioarqueológica desde el sitio Copaca 1. Chungara Revista de Antropología Chilena 48 (1):73-90.

Andrefsky, W. 1994. Raw material availability and the organization of technology. American Antiquity 59:21-35.

Andrefsky, W. 1998. Lithics: Macroscopic Approaches to Analysis. Cambridge Manuals in Archaeology. Cambridge University Press, Cambridge.

Andrus, C. 2011. Shell midden sclerochronology. Quaternary Science Reviews 30:2892-2906.

Arnold, J. 1996. The archaeology of Complex HunterGatherers. Journal of Archaeological Method and Theory 3 (2):77-126.

Báez, P., J. Arata y D. Jackson 2004. El Loco Concholepas concholepas (Bruguiere, 1789) (Mollusca: Gastropoda: Muricidae) como recurso durante el Holoceno Temprano-Medio en Los Vilos, Chile Central. Investigaciones Marinas 32 (1):107-113.

Ballester, B. 2017. La pesca y la caza marina en el desierto de Atacama: luces conceptuales desde los documentos escritos (siglos XVI-XIX). CUHSO 27 (2):89-120.

Ballester, B. 2018. Tecnología de arponaje en la costa del desierto de Atacama, norte de Chile. Estudios Atacameños 57:65-95.

Ballester, B., E. Calás, C. Pelegrino, E. Vidal y P. Aguilera 2017. La vida en comunidad de los cazadores-pescadores marinos del desierto de Atacama (4000-2000 cal. a.C.). En Monumentos Funerarios de la Costa del Desierto de Atacama: Contribuciones al Intercambio de Bienes e Información entre Cazadores-Recolectores Marinos (Norte de Chile), editado por F. Gallardo, B. Ballester y N. Fuenzalida, pp. 183-287. Centro Interdisciplinario de Estudios Interculturales e Indígenas, Sociedad Chilena de Arqueología. Sous Presse, Santiago.

Ballester, B. y A. Clarot 2014. La Gente de los Túmulos de Tierra. Marmot Impresores, Santiago.

Ballester B., A. Clarot y V. Bustos 2014a. Chacaya 2: reevaluación de un campamento Arcaico Tardío (6000 a la $4000 \mathrm{cal} \mathrm{AP)} \mathrm{de} \mathrm{la}$ costa de Mejillones, II región, Chile. Werkén 15:31-48.

Ballester, B., A. Clarot, V. Bustos, A. Llagostera y H. Garcés 2014b. Arqueología de la Prehistoria de la Península de Mejillones: el campamento de Los Canastos 3 desde sus cuadernos de campo y materiales de museo. Boletín de la Sociedad Chilena de Arqueología 43/44:5-21.

Ballester, B. y M. Crisóstomo 2017. Percutores líticos de la pampa del desierto de Atacama: Tecnología, huellas de uso, decoración y talladores. Chungara Revista de Antropología Chilena 49 (2):175-192.

Ballester, B. y F. Gallardo 2011. Prehistoric and historic networks on the Atacama Desert coast (northern Chile). Antiquity 85:875-889.

Ballester, B., E. Vidal, E. Calás, F. Gallardo, P. Aguilera, C. Pellegrino y A. Clarot 2018. Un enclave Arcaico Tardío en la aguada costera de Gualaguala (Desierto de Atacama, Norte de Chile). Chungara Revista de Antropología Chilena 50 (3):349-367.

Bate, L. 1971. Material lítico: metodología de clasificación. Noticiario Mensual del Museo Nacional de Historia Natural 181/182:3-24.

Béarez, P., F. Fuentes-Mucherl, S. Rebolledo, D. Salazar y L. Olguín 2016. Billfish foraging along the northern cast of Chile during the Middle Holocene (7400-5900 cal BP). Journal of Anthropological Archaeology 41:185-195.

Behrensmeyer, A. 1978. Taphonomic and Ecologic Information from Bone Weathering. Paleobiology 5:150-162.

Berenguer, J. 2009. Pescadores de la Niebla. Los Changos y sus Ancestros. Museo Chileno de Arte Precolombino, Santiago.

Binford, L. 1980. Willow smoke and dogs' tails: huntergatherer settlement systems and archaeological site formation. American Antiquity 45:4-10.

Binford, L. 1981. Bones: Ancient Men and Modern Myths. Academic Press, New York.

Binford, L. 1983. Long-term land use patterns: some implications for Archaeology. En Lulu Linear Punctated: Essays in Honor of George Irving Quimby, editado por R. Dunnell y D. Grayson, pp. 27-54. Anthropological Papers, Museum of Anthropology, University of Michigan, Michigan.

Binford, L. 1990. Mobility, housing, and environment: a comparative study. Journal of Anthropological Research 46 (2):119-152.

Binford, L. 2001. Constructing Frames of Reference: An Analytical Method for Archaeological Theory Building Using Hunter-Gatherer and Environmental Data Sets. University of California Press, Berkeley.

Binford, L. 2006. Bands as characteristic of "mobile huntergatherers" may exist only in the history of anthropology. En Archaeology and Ethnoarchaeology of Mobility, editado por F. Sellet, R. Greaves y P.L. Yu, pp. 3-22. University of Florida Press, Gainesville.

Bird, J. 1943. Excavations in Northern Chile. Anthropological Papers of the American Museum of Natural History 38 (4):173-318.

Bittmann, B. 1984. El Proyecto Cobija: investigaciones antropológicas en la costa del Desierto de Atacama (Chile). Actas del XLIV Congreso Internacional de Americanistas, pp. 99-146. Universidad del Norte, Antofagasta.

Bittman, B. y J. Munizaga 1984. Evolución en poblaciones precolombinas de la costa Norte de Chile. Chungara Revista de Antropología Chilena 13:129-142.

Blanco, J., M. De La Maza y Ch. Rees 2010. Cazadores recolectores costeros y el aprovisionamiento de recursos líticos. Perspectivas interpretativas de los eventos de talla del desierto absoluto. Werkén 13:45-68.

Borella, F., F. Grandi, D. Vales, N. Goodall y E. Crespo 2013. Esquema preliminar de fusión epifisaria en huesos de lobos marinos (Arctocephalus australis y Otaria avescens), su contribución en los análisis zooarqueológicos. En Tendencias Teórico-Metodológicas y Casos de Estudio en la Arqueología de la Patagonia, editado por A. Zangrando, R. Barberena, A. Gil, G. Neme, M. Giardina, L. Luna, C. Otaola, S. Paulides, L. Salgán y A. Tivoli, pp. 39-51. Museo de Historia Natural de San Rafael, Sociedad Argentina de Antropología e Instituto Nacional de Antropología y Pensamiento Latinoamericano, Buenos Aires.

Boisset, G., A. Llagostera y E. Salas 1969. Excavaciones arqueológicas en Caleta Abtao. Antofagasta. Actas del V Congreso Nacional de Arqueología Chilena, pp. 75-152. DIBAM, La Serena.

Borie, C., X. Power, S. Parra, H. Salinas, P. Rostan, P. Galarce, I. Peña y F. Traverso 2017. Tras la huella del sílice pampino. Nuevas metodologías para el rastreo de las áreas fuente de 
aprovisionamiento lítico en Taltal. Estudios Atacameños 56:103-131.

Borie, C., D. Salazar, X. Power, M. Figueroa, H. Orellana, S. Parra, C. Arenas, F. Traverso e I. Monroy 2018. Cazadores-recolectores marítimos en la Pampa Desértica de Taltal. Conocimientos, recursos, prácticas sociales y territorialización. En Estudios de Arqueología, Historia, Filosofia y Ciencias Sociales. En Homenaje a Mario Orellana Rodríguez (60 años de Vida Académica y Científica), editado por F. Orellana, pp. 205-242. Ediciones del Desierto, San Pedro de Atacama.

Borie, C. y C. Soto 2011. Prospecciones arqueológicas en la costa norte de Taltal. Taltalia 4:85-101.

Boyd, B. 2006. On 'sedentism' in the Later Epipaleolithic (Natufian) Levant. World Archaeology 38 (2):164-78.

Bronk Ramsey, C., y S. Lee 2013. Recent and planned developments of the program OxCal. Radiocarbon 55 (2-3):720-730.

Buchholtz E. y S. Schur 2004. Vertebral osteology in Delphinidae (Cetacea). Zoological Journal of the Linnean Society 140:383-401.

Burchell, M., A. Cannon, N. Hallmann, H. Schwarcz y B. Schöne 2013. Inter-site variability in the season of shellfish collection on the central coast of British Columbia. Journal of Archaeological Science 40:626-636.

Bustos, V. 1974. Chacaya II: una aldea temprana sin agricultura y sin cerámica. Arqueológica 2:12-41.

Camus, P., A. Navarrete, A. Sanhueza y L. Opazo 2012. Trophic ecology of the chiton Acanthopleura echinata on Chilean rocky shores. Revista Chilena de Historia Natural 85:123-135.

Capdeville, A. 1920. Diarios de Campo. Manuscrito inédito.

Capdeville, A. 1921a. Notas acerca de la arqueología de Taltal I. Boletín de la Academia Nacional de Historia 2 (3-4):1-23.

Capdeville, A. 1921b. Notas acerca de la arqueología de Taltal II: Civilización dolménica. Gentes de los círculos de piedra. Boletín de la Academia Nacional de la Historia 2 (5):1-13.

Castelleti, J. 2007. Patrón de Asentamiento y Uso de Recursos a través de la Secuencia Ocupacional Prehispana en la Costa de Taltal. Tesis para optar al grado de Magister en Antropología con mención en Arqueología. Universidad Católica del NorteUniversidad de Tarapacá, San Pedro de Atacama-Arica.

Castelleti, J. y G. Maltrain 2010. El formativo de Taltal y el patrón de asentamiento local. Actas del XVII Congreso de Arqueología Chilena Tomo 1, pp. 165-176. Ediciones Kultrún, Valdivia.

Castro, V., C. Aldunate y V. Varela 2012. Paisajes culturales de Cobija. Costa de Antofagasta, Chile. Revista Chilena de Antropología 26:97-128.

Castro, V., C. Aldunate, V. Varela, L. Olguín, P. Andrade, F. García-Albarido, F. Rubio, P. Castro, A. Maldonado y J. Ruz 2016. Ocupaciones arcaicas y probables evidencias de navegación temprana en la costa arreica de Antofagasta, Chile. Chungara Revista de Antropología Chilena 48 (4):503-530.

Castro, V., F. Maldonado y M. Vásquez 1993. Arquitectura en el Pukara de Turi. Actas del XII Congreso Nacional de Arqueología Chilena vol. 2:79-106. Boletín del Museo Regional de la Araucanía 4, Temuco.

Chatters, J. 1987. Hunter-gatherer adaptations and assemblage structure. Journal of Anthropological Archaeology 6:336-375.

Claassen, C. 1998. Shells. Cambridge University Press, New York.
Contreras, R. 2010. Recolección y pesca: pasado y presente en la costa de Taltal. Taltalia 3:57-86.

Contreras, R., J. Cruz, A. Llagostera, H. Garcés, P. Núñez, O. Rodríguez, H. Gárate y G. Becerra 2007. Los Bronces-1: un asentamiento de 5.500 años en la costa de Taltal. Fondo de Desarrollo Regional, Museo Augusto Capdeville Rojas, Taltal.

Contreras, R., J. Cruz, H. Garcés, A. Llagostera, P. Núñez, O. Rodríguez, G. Becerra y H. Gárate 2008. Los Bronces-1: un asentamiento de 5.500 años en la costa de Taltal. Taltalia 1:61-74.

Contreras, R., P. Núñez, A. Llagostera, J. Cruz, A. San Francisco, B. Ballester, O. Rodríguez y G. Becerra 2011. Un conglomerado del período Arcaico costero medio del área Taltal Paposo, Norte de Chile. Taltalia 4:7-31.

Cruz, J. y A. Llagostera 2011. Prehistoria de Antofagasta. En la Ruta de los Primeros Antofagastinos. Morgan impresores, Antofagasta.

De Souza, P., I. Cartajena, L. Núñez y C. Carrasco 2010. Cazadores-recolectores del Arcaico Tardío y desarrollo de complejidad social en la puna de Atacama: las evidencias del sitio Tulán-52 (norte árido de Chile). Werkén 13:91-118.

De Souza, P., C. Sinclaire, R. Molina y F. Gallardo 2002. Una nota sobre obsidianas de una fuente secundaria en la quebrada Pelún (localidad de Machuca, San Pedro de Atacama). Boletín de la Sociedad Chilena de Arqueología 33/34:81-83.

Diehl, M. 1992. Architecture as a material correlate of mobility strategies: some implications for archaeological interpretation. Behavior Science Research 26:1-35.

Durán, A. 1981. Investigación Arqueológica en Punta Grande. Memoria para optar al Título de Arqueólogo. Universidad del Norte, Antofagasta.

Durán, A. 1985. Algunos problemas en relación a las ocupaciones con anzuelo de concha en la zona de Taltal. Actas del IX Congreso Nacional de Arqueología Chilena, pp. 105-125. Museo Arqueológico de La Serena, La Serena.

Escobar, J. 2017. Caza tradicional de la albacora. Imaginario y patrimonio intangible de los pescadores de Taltal. Taltalia 10:69-85.

Falabella, F., R. Meléndez y L. Vargas 1995. Claves Osteológicas para Peces de Chile Central: un Enfoque Arqueológico. Artegrama Ltda, Santiago.

Figueroa, M. 2007. Análisis Lítico del sitio Óvalo Le Paige(II Región). Informe de práctica profesional en Arqueología, Departamento de Antropología, Facultad de Ciencias Sociales, Universidad de Chile, Santiago.

Fitzhugh, B., S. Colby Phillips y E. Gjesfjeld 2011. Modeling hunter-gatherer information networks: an archaeological case study from Kuril Islands. En Information and its Role in HunterGatherer Bands, editado por R. Whallon, W. Lovis y R. Hitchcock, pp. 85-166. Ideas, Debates, and Perspectives 5, Cotsen Institute of Archaeology Press, Los Angeles.

Flores, C., V. Figueroa y D. Salazar 2016. Middle Holocene production of mussel shell fishing artifacts on the coast of Taltal (25 Lat South), Atacama Desert, Chile. The Journal of Island and Coastal Archaeology 11:411-424.

Gaete, N., X. Navarro, M. Vargas, S. Morales, M. Uribe, H. Velásquez, B. Ladrón De Guevara, S. Teillier, M. García, A. Román, L. Quiroz, A. Sáez y M. Godoy 2003. Plan de rescate arqueológico Ruta 1 sector Taltal-Punta Viento, Comuna de Taltal, Provincia de Antofagasta, II Región. Sitio 02 Ta 007 "Morro Colorado". Informe de Investigación. Convenio MOP-CMT. Consejo de Monumentos Nacionales, Chile. 
Galarce, P. y G. Santander 2013. Contextos líticos de asentamientos arcaicos en la costa de Taltal (II Región, Chile). Estudios Atacameños 46:5-26.

Gamble, C. 1983. Culture and society in the upper Paleolithic Europe. En Hunter Gatherer Economy in Prehistory, editado por Bailey, G., pp. 201-211. Cambridge University Press, Cambridge.

García-Albarido, F. 2012. Estrategias de subsistencia en Cobija durante el periodo Intermedio Tardio y el Tawantinsuyu. Aproximación desde la Funcionalidad de los Instrumentos Líticos. Tesis para optar al título de Arqueólogo, Departamento de Antropología, Universidad de Chile, Santiago.

González, B., R. Palma, B. Zapata y J. Marín 2006. Taxonomic and biogeographical status of guanaco Lama guanicoe (Artiodactyla, Camelidae). Mammal Review 36 (2):157-178.

Gould, R. y S. Saggers 1985. Lithic procurement in Central Australia: a closer look at Binford's idea of embeddedness in archaeology. American Antiquity 50 (1):117-136.

Grayson, D. 1984. Quantitative Zooarchaeology. Academic Press, Orlando.

Guendon, J. 2009. Informe Geomorfológico. Proyecto FONDECYT 1080666. Manuscrito en posesión del autor.

Guráieb, A. 2001. La agregación en cazadores-recolectores: aportes desde la etnografía y la arqueología. Relaciones de la Sociedad Argentina de Antropología XXVI:369-383.

Gutiérrez, G. y L. Lazo 1996. Plantas Medicinales Silvestres de Uso Tradicional en la Localidad de Paposo, Costa del Desierto de Atacama, II Región, Chile. Fondo de Desarrollo de las Artes y la Cultura, Ministerio de Educación, Santiago.

Guzmán, N., S. Saa y L. Ortlieb 1998. Catálogo descriptivo de los moluscos litorales (Gastropoda y Pelecipoda) de la zona de Antofagasta, $23^{\circ} \mathrm{S}$ (Chile). Estudios Oceanológicos 17:17- 86.

Herrera, C. y E. Custodio 2014. Origin of waters from small springs located at the northern coast of Chile, in the vicinity of Antofagasta. Andean Geology 41 (2):314-341.

Herrera, C., C. Gamboa, E. Custodio, T. Jordan, L. Godfrey, J. Jódar, J. Luque, J. Vargas y A. Sáez 2018. Groundwater origin and recharge in the hyperarid Cordillera de la Costa, Atacama Desert, northern Chile. Science of the Total Environment 624:114-132.

Hitchcock, R. y J. Ebert 2011. Where is that job? Huntergatherers information systems in complex social environments in the easter Kalahari Desert, Botswana. En Information and its Role in Hunter-Gatherer Bands, editado por R. Whallon, W. Lovis y R. Hitchcock, pp. 133-166. Ideas, Debates, and Perspectives 5, Cotsen Institute of Archaeology Press, Los Angeles.

Hogg, A., Q. Hua, P. Blackwell, M. Niu, M., C. Buck, T. Guilderson, T. Heaton, J. Palmer, P. Reimer, R. Reimer, C. Turney y S. Zimmerman 2013. SHCal13 Southern Hemisphere Calibration, 0-50,000 Years cal BP. Radiocarbon 55 (4):18891903.

Jerardino, A., J. Castilla, J. Ramírez y N. Hermosilla 1992. Early coastal subsistence patterns in central Chile: a systematic study of the marine-invertebrate fauna from the site of Curaumilla-1. Latin American Antiquity 3 (1):43- 62.

Kasuya, T. 1973. Systematic consideration of recent toothed whales based on the morphology of tympano-periotic bone. The Scientific Reports of the Whales Research Institute 25:1-103.

Kaufmann, C. 2004. La fusión ósea como indicador de edad y estacionalidad en guanaco (Lama guanicoe). En Contra
Viento y Marea. Arqueología de la Patagonia, editado por M.T. Civalero, P.M. Fernández y A.G. Guráieb, pp. 477-487. Instituto Nacional de Antropología y Pensamiento Latinoamericano y Sociedad Argentina de Antropología, Buenos Aires.

Keeley, L. 1982. Hafting and retooling: effects on the archaeological record. American Antiquity 47:798-809.

Kelly, R. 1983. Hunter-gatherer mobility strategies. Journal of Anthropological Research 39:277-306.

Kelly, R. 1992. Mobility/ sedentism: concepts, archaeological measures and effects. Annual Review of Anthropology 21:43-66.

Kelly, R. 1995. The Foraging Spectrum: Diversity in Hunter-Gatherers Lifeways. Smithsonian Institution Press, Washington, DC.

Kelly, R., L. Poyer y B. Tucker 2006. Mobility and houses in Southwestern Madagascar: ethnoarchaeology among the Mikea and their neighbors. En Archaeology and Ethnoarchaeology of Mobility, editado por F. Sellet, R. Greaves y P.L. Yu, pp. 75107. University of Florida Press, Gainesville.

Labarca, R., E. Calás y A. Prieto 2017. Los artefactos óseos de contextos funerarios costeros de la región de Antofagasta. En Monumentos Funerarios de la Costa del Desierto de Atacama: Contribuciones al Intercambio de Bienes e Información entre Cazadores-Recolectores Marinos (Norte de Chile), editado por F. Gallardo, B. Ballester y N. Fuenzalida, pp. 95-106. Centro Interdisciplinario de Estudios Interculturales e Indígenas, Sociedad Chilena de Arqueología, Sous Presse, Santiago.

Lamilla, J. y C. Bustamante 2005. Guía para el reconocimiento de: tiburones, rayas y quimeras de Chile. Oceana 17:1-80.

Latcham, R. 1939. La Edad de Piedra en Taltal. Boletín del Museo Nacional de Historia Natural XVII:1-47.

Lazzari, M. 2005. Traveling objects and spatial images: exchange relationships and the production of social space. En Global Archaeological Theory: Contextual Voices and Contemporary Thoughts, editado por P.P.A. Funari, A. Zarankin y E. Stovel, pp. 191-210. Kluwer Academic/Plenum Publisher, New York.

Llagostera, A. 1989. Caza y pesca marítima (9000 a 1000 a.C.). En Prehistoria. Desde sus Orígenes hasta los Albores de la Conquista, editado por J. Hidalgo, V. Schiappacasse, H. Niemeyer, C. Aldunate e I. Solimano, pp. 57-79. Editorial Andrés Bello, Santiago.

Llagostera, A. 1990. La navegación prehispánica en el Norte de Chile: bioindicadores e inferencias teóricas. Chungara Revista de Antropología Chilena 24/25:37-51.

Llagostera, A. 2005. Culturas costeras precolombinas en el norte chileno: secuencia y subsistencia de las poblaciones arcaicas. En Biodiversidad Marina: Valoración, Usos, Perspectivas ¿Hacia Dónde va Chile?, editado por E. Figueroa, pp. 107-148. Editorial Universitaria, Santiago.

Llagostera, A. 2013. Poblaciones marítimas con arquitectura. Hombre y Desierto 17:150-182.

Llagostera, A y V. Llagostera 2010. Enterratorios del sitio arqueológico Los Bronces-1, Comuna de Taltal (Región de Antofagasta). Taltalia 3:7-20.

Luedtke, B. 1992. An Archaeologist's Guide to Chert and Flint. Archaeological Research Tools 7. UCLA, Institute of Archaeology, Los Angeles.

Lyman, R. 2008. Quantitative Paleozoology. Cambridge University Press, New York.

Macdonald, D. y B. Hewlett 1999. Reproductive interest and forager mobility. Current Anthopology 40 (4):501-523. 
MacEachern, S. 1994. "Simbolic reservoirs" and inter-group relations: West African examples. The African Archaeological Review 2:205-224.

Maher, L. y M. Conkey 2019. Homes for hunters? Exploring the concept of home at hunter-gatherer sites in Upper Paleolithic Europe and Epipaleolithic Southwest Asia. Current Anthropology 60 (1):91-137.

Marincovich, L. 1973. Intertidal Mollusks of Iquique, Chile. Natural History Museum, Los Angeles County, Science Bulletin 16:1-49

Marquet, P., F. Bozinovic, G. Bradshaw, C. Cornelius, H. González, J. Gutierrez, E. Hajek, J. Lagos, F. López-Cortés, L. Núñez, E. Rosello, C. Santoro, H. Samaniego, V. Standen, J. Torres-Mura y F. Jaksic 1998. Los ecosistemas del desierto de Atacama y área andina adyacente en el norte de Chile. Revista Chilena de Historia Natural 71:593-617.

Marshall, Y. 2006. Introduction: adopting a sedentary lifeway. World Archaeology 38 (2):153-163.

Martínez, K. 2008. Caracterización Morfológica y Métrica del Hueso Periótico del Suborden Odontoceti con Registro dentro del Territorio Chileno. Memoria para optar al título profesional de Médico Veterinario, Departamento de Ciencias Biológicas Animales, Universidad de Chile, Santiago.

Mason, R., M. Peterson y J. Tiffany 1998. Weighing vs. counting: measurement reliability and the California school of midden analysis. American Antiquity 63:303-324.

Mengozzi, F. 2016. Hacia una aproximación de las Tradiciones Tecnológicas de Cuentas de las "Poblaciones Marítimas con Arquitectura” en la Costa Arreica del Norte Grande de Chile. Tesis para optar al título profesional de Arqueóloga, Departamento de Antropología, Universidad de Chile, Santiago.

Miotti, L. y L. Marchionni 2014. Autopodios de guanacos en sitios arqueológicos: equinalidad entre lo palatable y lo preservado. Revista Chilena de Antropología 29:122-129.

Monks, G. 1981. Seasonality studies. En Advances in Archaeological Method and Theory Vol. 4. editado por M. Schiffer, pp. 177-240. Academic Press, New York.

Montenegro, N. 1981. Excavación y Estudio del Sitio Arqueológico Punta Guasilla: Un Asentamiento Temprano. Cobija, Tocopilla. Memoria para optar al Título de Arqueóloga, Universidad del Norte, Antofagasta.

Mostny, G. 1964. Arqueología de Taltal. Epistolario de Augusto Capdeville con Max Uhle y otros. Fondo Histórico y Bibliográfico José Toribio Medina, Santiago.

Nakamura, I. 1985. Billfishes of the world. An annotated and illustrated catalogue of marlins, sailfishes, spearfishes and swordfishes known to date. FAO Fisheries Synopsis 125 (5):1-65.

Nelson, M. 1991. The study of technological organization. Archaeological Method and Theory 3:57-100.

Núñez, L. 1971. Secuencia y cambio en los asentamientos humanos de la desembocadura del río Loa en el Norte de Chile. Boletín de la Universidad de Chile 112:3-25.

Núñez, L. 1984. Secuencia de asentamientos prehistóricos del área de Taltal. Revista Futuro 8:28-76. Departamento Municipal Escolar, Ilustre Municipalidad de Taltal, Taltal.

Núñez, L. y C. Perlès 2018. Ceremonialismo y congregación durante la transición Arcaico Tardío y Formativo Temprano en la circumpuna de Atacama (norte de Chile). Revista de Geografía Norte Grande 70:183-209.
Núñez, L y C. Santoro 2011. El tránsito Arcaico-Formativo en la circumpuna y valles occidentales del centro sur andino: hacia los cambios "neolíticos". Chungara Revista de Antropología Chilena 43:487-531.

Núñez, L. y J. Varela 1967-68. Sobre los recursos de agua y el poblamiento prehispánico de la costa del Norte Grande de Chile. Estudios Arqueológicos 3/4:7-41.

Núñez, L., V. Zlatar y P. Núñez 1974. Caleta Huelén 42. Una Aldea Temprana en el Norte de Chile (Nota Preliminar). Imprenta Universitaria, Universidad de Panamá, Panamá.

Núñez, P. 1974. Notas sobre la aldea preagrícola Caleta Huelén 42, desembocadura del río Loa. Norte de Chile. Serie Documentos de Trabajo 5:27-43.

Olguín, L. 2013. Aprovechamiento de invertebrados marinos en conchales arqueológicos del Arcaico Medio (6.000-4.000 B.C) en la costa de Taltal: estudios preliminares. Taltalia 5-6:37-53.

Olguín, L. 2014. El rol económico de los recursos malacológicos durante el Holoceno Medio en la costa arreica del Norte de Chile: sitio Agua Dulce. Revista Chilena de Antropología 29:32-39.

Olguín, L., D. Salazar y D. Jackson 2014. Tempranas evidencias de navegación y caza de especies oceánicas en la costa pacífica de Sudamérica (Taltal, 7.000 años cal. a.p.) Chungara Revista de Antropología Chilena 46 (2):177-192.

Olguín, L., V. Castro, P. Castro, I. Peña-Villalobos, J. Ruz y B. Santander 2015a. Exploitation of faunal resources by marine hunter gatherer groups during the Middle Holocene at the Copaca 1 site, Atacama Desert coast. Quaternary International 373:4-16.

Olguín, L., C. Flores, C., y D. Salazar 2015b. Aprovechamiento humano de moluscos marinos en conchales arqueológicos del Holoceno Temprano y Medio (12.000- 5.500 años cal. AP), costa meridional del desierto de Atacama, Chile. En Arqueomalacología: Abordajes Metodológicos y Casos de Estudios en el Cono Sur, editado por H. Hammond y M. Zubimendi, pp. 13-34. Fundación de Historia Natural Félix de Azara, Buenos Aires.

Ortlieb, L., G. Vargas y J. Saliége 2010. Marine radiocarbon reservoir effect along the northern Chile- southern Peru coast (14-24 $\left.{ }^{\circ} \mathrm{S}\right)$ throughout the Holocene. Quaternary Research 75:91-103.

Osorio, C. 2002. Moluscos Marinos en Chile: Especies de Importancia Económica. Guía para su identificación. Facultad de Ciencias, Universidad de Chile, Santiago.

Otaiza, R. y B. Santelices 1975. Vertical distribution of chitons (Mollusca: Polyplacophora) in the rocky intertidal zone of central Chile. Journal Experimental Marine Biology and Ecology 86:229-240.

Pacheco-Torres, V., A. Altamirano y E. Guerra 1986. The Osteology of South American Camelids. Institute of Archaeology University of California, Los Angeles.

Pavés, H., R. Schlatter y C. Espinoza 2005. Patrones reproductivos del lobo marino común, Otaria flavescens (Shaw, 1800), en el centro-sur de Chile. Revista Chilena de Historia Natural 78:687-700.

Pimentel, G., C. Rees, P. De Souza y L. Arancibia 2011. Viajeros costeros y caravaneros. Dos estrategias de movilidad en el Período Formativo del Desierto de Atacama, Chile. En En Ruta. Arqueología, Historia y Etnografía del Tráfico Sur Andino, editado por L. Núñez y A. Nielsen, pp. 43-81. Encuentro Grupo Editor, Córdoba. 
Pimentel, G. y M. Ugarte 2017. La agencia costera en la pampa del Desierto de Atacama. En Monumentos Funerarios de la Costa del Desierto de Atacama: Contribuciones al Intercambio de Bienes e Información entre Cazadores-recolectores Marinos (Norte de Chile), editado por F. Gallardo, B. Ballester y N. Fuenzalida, pp. 2536. Centro Interdisciplinario de Estudios Interculturales e Indígenas, Sociedad Chilena de Arqueología, Sous Presse, Santiago.

Pimentel, G., M. Ugarte, J. Blanco, C. Torres-Rouff y W. Pestle 2017. Calate: de lugar desnudo a laboratorio arqueológico de la movilidad y el tráfico intercultural prehispánico en el desierto de Atacama (ca. 7000 AP-550 AP). Estudios Atacameños 56:21-56.

Politis, G. 2006. The different dimensions of mobility among the Nukak foragers of the Colombian Amazon. En Archaeology and Ethnoarchaeology of Mobility, editado por F. Sellet, R. Greaves y P. L. Yu, pp. 23-43. University of Florida Press, Gainesville.

Power, X. 2015. Función y Estructura del Sitio Caleta Bandurrias (Taltal, II Región de Antofagasta). Una Evaluación sobre las Poblaciones Costeras de los "Círculos De Piedra". Tesis para optar al título profesional de Arqueóloga, Departamento de Antropología, Universidad de Chile, Santiago.

Power, X. 2017. Propuesta Teórico-Metodológica para el Estudio Intrasitio de Yacimientos del Arcaico Tardío de Taltal, Norte de Chile. Tesis para optar al grado de Magíster en Antropología. Universidad Católica del Norte-Universidad de Tarapacá, San Pedro de Atacama-Arica.

Raedeke, K. y J. Simonetti 1988. Food habits of Lama guanicoe in Atacama Desert of northern Chile. Journal of Mammalogy 69:198-201.

Rebolledo, S., P. Béarez, D. Salazar y F. Fuentes 2016. Maritime fishing during the Middle Holocene in the hyperarid coast of the Atacama Desert. Quaternary International 391:3-11.

Reise, D. 1973. Clave para la determinación de los cráneos de marsupiales y roedores chilenos. Gayana Zoología 27:1-20.

Salazar, D., C. Palma, H. Salinas, F. Fuentes, J. Guendon y C. Castellón 2010. Reconstrucción de la secuencia ocupacional de la quebrada Mamilla, costa de Tocopilla, Norte de Chile. Werkén 13:323-346.

Salazar D., D. Jackson, J. Guendon, H. Salinas, D. Morata, V. Figueroa, G. Manríquez y V. Castro 2011. Early Evidence (ca. 12,000 B.P.) for Iron Oxide Mining in the Pacific Coast of South America. Current Anthopology 52 (5):463-75.

Salazar, D., V. Figueroa, P. Andrade, H. Salinas, L. Olguín, X. Power, S. Rebolledo, S. Parra, H. Orellana y J. Urrea 2015. Cronología y organización económica de las poblaciones arcaicas de la costa de Taltal. Estudios Atacameños 50:7-46.

Santander, B. 2011. Patrones de Huellas de Uso en Artefactos Óseos para el Período Formativo Temprano en la Puna de Atacama. El Sitio Tulan-54. Memoria para optar al título profesional de Arqueólogo, Departamento de Antropología, Universidad de Chile, Santiago.

Schaedel, R. 1957. Arqueología Chilena. Contribuciones al Estudio de la Región Comprendida entre Arica y La Serena. Centro de Estudios Antropológicos, Universidad de Chile, Santiago.

Schlanger, S. 1992. Recognizing persistent places in Anasazi settlement systems. En Space, Time and Archaeological Landscapes, editado por J. Rossignol y L. Wandsnider, pp. 91112. Plenum Press, New York.

Seelenfreund, A., C. Sinclaire, M. Dinator, J. Morales, D. Pasten, P. Miranda, S. Cancino, L. Lera y Ch. Rees 2010. Negras y brillantes: explotación y distribución de obsidianas en la macro región del Salar de Atacama. Actas del XVII Congreso Nacional De Arqueología Chilena Tomo II, pp. 813824. Ediciones Kultrún, Valdivia.

Sielfeld, W. 1999. Estado del conocimiento sobre la conservación y preservación de Otaria flavescens (Shaw, 1800) y Arctocephalus australis (Zimmermann, 1783) en las costas de Chile. Estudios Oceanológicos 18:81-96.

Sierpe, V. 2015. Atlas Osteológico del Guanaco (Lama guanicoe). Ediciones Universidad de Magallanes, Punta Arenas.

Silva, J. y D. Bahamondes 1969. Investigaciones arqueológicas en Taltal. Informe Preliminar. Actas del IV Congreso Nacional de Arqueología, Rehue 2:7-25.

Smith, C. 2003. Hunter-gatherer mobility, storage, and houses in a marginal environment: an example from the Mid-Holocene of Wyoming. Journal of Anthropological Archaeology 22:162-189.

Soto, C., X. Power y B. Ballester 2018. Circulación de objetos perforados de concha: aportes para la interpretación de su rol en las relaciones sociales del Desierto de Atacama entre los 60003500 años Cal AP. Boletín del Museo de Arte Precolombino 23 (1):53-71.

Standen, V. y B. Arriaza 2016. Cultura Chinchorro. Catálogo de Artefactos. Ediciones Universidad de Tarapacá, Arica.

Stein, J., J. Deo y L. Phillips 2003. Big sites-short time: accumulation rates in archaeological sites. Journal of Archaeological Science 30:297-316.

Taylor, W. 1964. Tethered nomadism and water territoriality: an hypothesis. Actas del XXXV Congreso Internacional de Americanistas 2: 197-203. Instituto Nacional de Antropología e Historia, México, DF.

Thiel, M., E. Macaya, E. Acuña, W. Arntz, H. Bastías, K. Brokordt, P. Camus, J. Castilla, L. Castro, M. Cortés, C. Dumont, R. Escribano, M. Fernández, J. Gajardo, C. Gaymer, I. Gómez, A. González, H. González, P. Haye, J. Illanes, J. Iriarte, D. Lancellotti, G. LunaJorquera, C. Luxoro, P. Manríquez, V. Marín, P. Muñoz, S. Navarrete, E. Pérez, E. Poulin, J. Sellanes, H. Sepúlveda, W. Stotz, F. Tala, A. Thomas, C. Vargas, J. Vásquez y J. Vega 2007. The Humboldt Current System of northern and central Chile: oceanographic processes, ecological interactions and socioeconomic feedback. Oceanography and Marine Biology 45:195-344.

Urbina, S., L. Adán, C. Moragas, S. Olmos y R. Ajata 2011. Arquitectura de asentamientos de la costa de Tarapacá, norte de Chile. Estudios Atacameños 41:63-96.

Urrejola, C. y M. Orellana 2000. Explotación de recursos líticos en la zona desértica al interior de Taltal. Libro Resúmenes del XV Congreso Nacional de Arqueología Chilena, pp. 79. Arica.

Varela, V. 2009. La cerámica arqueológica de Taltal. Taltalia 2:119-128.

Wandsnider, L. 1992 The spatial dimension of time. In Space, Time, and Archaeological Landscapes, editado por J. Rossignol y L. Wandsnider, pp. 257-282. Plenum Press, New York.

Waselkov, G. 1987. Shellfish gathering and shell midden archaeology. En Advances in Archaeological Method and Theory Vol. 10, editado por M. Schiffer, pp. 257-310. Academic Press, New York.

Whallon, R., 2006. Social networks and information: non"utilitarian" mobility among hunter gatherers. Journal of Anthropological Archaeology 25:259-270.

Whallon, R., W.A. Lovis y R.K. Hitchcock 2011. Information and its Role in Hunter-Gatherer Bands. Cotsen Institute of Archaeology Press, UCLA, Los Angeles, California. 
Wiessner, P. 1982. Beyond willow smoke and dogs' tails: a comment on Binford's analysis of hunter-gatherer settlement systems. American Antiquity 47:171-177.

Wiessner, P. 1983. Style and Social Information in Kalahari San Projectile Points. American Antiquity 48 (2):253-76.

Wobst, H. 1974. Boundary conditions for Paleolithic social systems: a simulation approach. American Antiquity 39:147-179.

Wobst, H. 2006. Artifacts as social interference: the politics of spatial scale. En Confronting Scale in Archaeology: Issues of Theory and Practice, editado por G. Lock y B.L. Molyneaux, pp. 55-64. Springer, New York.
Yesner, D. 1980. Maritime Hunter-Gatherers: Ecology and Prehistory. Current Anthropology 21 (6):727-735.

Zlatar, V. 1983. Replanteamiento sobre el problema Caleta Huelén 42. Chungara Revista de Antropología Chilena 10:2128.

Zlatar, V. 1987. Un yacimiento precerámico y su problemática desde la perspectiva de sus recintos habitacionales. Hombre $y$ Desierto 1:1-36.

Zúñiga, O. 2002. Moluscos: Guía de Biodiversidad. CREA, Universidad de Antofagasta, Antofagasta.

\section{Notas}

${ }^{1}$ Aún cuando no se registra un contexto mortuorio stricto sensu, se infiere el uso funerario de la Estructura-1 a partir de la asociación de restos humanos, colorantes minerales y artefactos característicos de los ajuares funerarios de sitios afines (Andrade et al. 2016; Ballester et al. 2014b, 2014a; 2018; Capdeville 1921b; Castro et al. 2016; Contreras et al. 2007, 2008; Llagostera y Llagostera 2011; Mostny 1964; Núñez 1984). El malogrado estado de conservación de los restos bioantropológicos sólo permitió la identificación de un calcáneo y un talus, a la vez que de algunos huesos largos no diferenciados (Andrade com. pers. 2019).

${ }^{2}$ La presencia de autopodios de camélidos en sitios arqueológicos plantea un problema de equifinalidad entre la selectividad antrópica (retorno económico) y su alto índice de preservación (cf. Miotti y Marchionni 2014).

${ }^{3}$ Recientes estudios señalan que las cuentas líticas constituyen piezas únicas o minoritarias en los colgantes registrados en los ajuares de este periodo. Igualmente, presentan características morfométricas disímiles a las conspicuas piezas de concha que conforman dichos complementos ornamentales (Mengozzi 2016).

${ }^{4}$ Una prospección intensiva de la puntilla demostró la presencia de numerosos loci de extracción y procesamiento de recursos específicos, sugierendo actividades vinculadas al radio de forrajeo diario respecto al asentamiento principal.

${ }^{5} \mathrm{Si}$ bien indispensables para el aparataje tecnológico y el rango de vida de los grupos cazadores recolectores pescadores de estudio, los viajes a larga distancia con fines de procurar materias primas o intercambio, no se consideran aquí variables definitorias de los movimientos residenciales (cf. Kelly 1983, 1995; Gould y Saggers 1985).

${ }^{6}$ Adicionalmente se reportan numerosos sitios con arquitectura de patrón Caleta Huelén 42 sin estudios específicos, como son: Chipana (Núñez et al. 1974) y Caleta Huelén-48 y 75 en la desembocadura del Río Loa (Ballester et al. 2017; Núñez 1971); Agua Dulce, Rocas Negras, Paraguas y Punta Guanillos en Tocopilla (Salazar et al. 2010); Caleta Las Tórtolas, Caleta Oliva, Agua de los Perros, Caleta de las Guaneras, Caleta Peña Blanca, El Gritón, Atacama-1 y San Pedro-1 y 2 (Ballester y Gallardo 2011; Ballester et al. 2017; Contreras et al. 2007; Latcham 1939; Núñez y Santoro 2011) en Taltal.

${ }^{7}$ Otra locación extractiva operando en el marco del radio forrajero litoral durante este momento, corresponde a San Ramon-15, la cual evidencia una explotación minera de baja intensidad y sin señal de actividad doméstica (Salazar et al. 2011). 
